\title{
Nonlinear stability in the spatial attitude motion of a satellite in a circular orbit
}

\author{
Daniela Cárcamo-Díaz • Jesús F. \\ Palacián · Claudio Vidal · Patricia \\ Yanguas
}

Received: date / Accepted: date

\begin{abstract}
The paper considers the attitude nonlinear stability analysis of the spatial satellite problem and takes it one step further. A study of the Lie (formal) stability is presented and long-time estimates related to the Lie stable cases are provided. The connection with Nekhoroshev theory is also shown. Finally, KAM tori related to Lie stable, as well as unstable equilibria, are also calculated.
\end{abstract}

Keywords Spatial satellite - Attitude - Resonances · Formal and Lie stability $\cdot$ Exponential estimates

PACS PACS 02.30.Hq · PACS 45.50.Pk

Mathematics Subject Classification (2000) 37J25 - 37N05 - 70F15 . $70 \mathrm{~K} 28 \cdot 65 \mathrm{~L} 70$

\section{Introduction}

The interest of studying the nonlinear stability of the satellite problem is due to the importance of astrodynamics projects related to putting satellites into orbit. Attitude control provides information about the satellite's orientation

The authors are partially supported by Project MTM 2017-88137-C2-1-P of the Ministry of Science, Innovation and Universities of Spain. D. Cárcamo-Díaz acknowledges support from CONICYT PhD/2016-21161143. C. Vidal is partially supported by Fondecyt grant 1180288.

D. Cárcamo-Díaz, C. Vidal

Grupo de Investigación en Sistemas Dinámicos y Aplicaciones-GISDA, Departamento de Matemática, Facultad de Ciencias, Universidad del Bío-Bío, Concepción, VIII Región, Chile E-mail: danielacarcamodiaz@gmail.com, clvidal@ubiobio.cl

J.F. Palacián, P. Yanguas

Departamento de Estadística, Informática y Matemáticas and Institute for Advanced Materials and Mathematics (INAMAT ${ }^{2}$ ), Universidad Pública de Navarra, 31006 Pamplona, Spain

E-mail: \{palacian, yanguas\}@unavarra.es 
with respect to a reference system and its precise guidance, which includes attitude stabilization. This has been a fundamental problem since the beginning of the Space Age and therefore, there is a vast literature on the attitude stability of a satellite in a circular orbit in a central gravity field. A beautiful historical introduction on the study of the librational stability of satellites can be found in [21]. Here we only mention a few papers. For a basic reference see for instance, [19].

The linear stability of the satellite problem was studied by Beletskii [3], [4], Delp [11] and DeBra and Delp in [10]. They found that in the plane of parameters defined by the quotients of the satellite's principal moments of inertia there are two regions, I and II, where the problem's equilibria are linearly stable. In fact, in region I there is Liapunov stability, thus nonlinear stability, whereas in region II the conditions for stability are necessary but not sufficient. In the literature, region I is known as Lagrange region and II is called Beletskii-DeBra-Delp region. In [24] we find the first nonlinear stability analysis in region II. Certain non-resonant and single resonant cases are inspected. Some of them are found to be Liapunov stable, others are formally stable, further ones are stable for the majority of initial conditions and others are unstable. In [31] and [32], Zhavnerchik determines the instability in a certain double resonant situation. Generalisations of this problem have been widely considered in the literature, see for instance, the elliptical case [2].

Lie stability of equilibria is a special case of formal stability of Siegel [30] and Moser [27]. It was dubbed Birkhoff stability in the pioneer work by Khazin [20]. This concept appears as a strong alternative in cases where Liapunov stability cannot be ensured [6], [7] and one requires a sort of nonlinear stability. Moreover, Lie stability is obtained for Hamiltonian systems that do not satisfy Nekhoroshev's theory necessary conditions. For example we find degenerate cases that do not comply with directional quasi-convexity or cases where stability is decided by the resonant terms [8]. The determination of Lie stability passes through obtaining the required convexity of the Hamiltonian function restricted to a subspace that we will call $S$ and that is contained in the orthogonal space related to the frequency vector. We recall the definition of $S$, as well as other basics on Lie stability, in Appendix 7 .

In the Lie stable cases, the error estimates of the solutions over exponentially long times are obtained through a result based in the determination of error bounds for adiabatic invariants in Hamiltonian systems [9]. The theorem appears for the first time in [7], see also [6]. We recall it in Appendix 7 and particularise it for our system in Sect. 4.

The paper is structured as follows. In Sect. 2 we establish the equations of motion of the system, the equilibria and the regions where linear stability is accomplished. The main results of the manuscript are contained in Sect. 3 and Sect. 4, the first one including our analysis on the Lie stability of the satellite's attitude dynamics. Sect. 4 presents the asymptotic estimates on the solutions in the Lie stable cases. In Sect. 5 we establish the existence of invariant 3tori encasing the quasi-periodic solutions corresponding to the equilibria. In Sect. 6 we outline some conclusions. At the end of the paper there are three 
appendices, respectively, Sects. 7, 8 and 9. The first one is devoted to recall the concepts and central theorems used to establish Lie stability and give estimates on the solutions. Readers not familiar with Lie stability are recommended to go through this section before getting immersed in Sect. 3. Some useful tables with coefficients lists appear in the second appendix. Finally, Sect. 9 applies Nekhoroshev theory for the study of the attitude stability.

The main contributions of our approach are Theorem 1, where we establish the Lie stability of the attitude dynamics in terms of two external parameters, which are the ratios of the satellite's principal moments of inertia, and Theorem 2 where asymptotic bounds for the Lie stable cases are provided. Finally, we also establish Theorem 3, that deals with the existence of 3-dimensional KAM tori and quasi-periodic motions associated to the Lie stable equilibria and also a case of an unstable equilibrium.

\section{Setting of the problem}

We consider the motion of a satellite with respect to its centre of mass in a central gravitational field. The centre of mass describes a circular orbit and the satellite has non-necessarily equal principal central moments of inertia, namely, $a, b$ and $c$. For more details the reader is addressed to [24] or [23] and references therein.

Let $\mathrm{O} u v w$ be a coordinate system whose origin coincides with the centre of mass of the satellite and whose axes are directed along the principal central axes of the satellite's ellipsoid of inertia. Its position relative to the orbital coordinate system $\mathrm{O} U V W$ (the $\mathrm{O} U$ axis is directed along the radius vector of the centre of mass, $\mathrm{OV}$ is along the transversal, and $\mathrm{O} W$ is along the normal to the plane of the orbit) is specified by means of the Euler angles $x$, $y$ and $z$, see Fig. 1 . The momenta conjugate to $(x, y, z)$ are named $(X, Y, Z)$. According to [24], [23] the relative motion of the satellite can be described by the canonical system of differential equations associated to the autonomous Hamiltonian function with three degrees of freedom

$$
\begin{aligned}
H= & \frac{1}{2} Y^{2}\left(\frac{A}{C}+\frac{A \cos ^{2} y+\sin ^{2} y}{\tan ^{2} x}\right)+\frac{1}{2}(A-1) X Y \cot x \sin (2 y) \\
& +\frac{3(C-1)}{2 A} \sin ^{2} x \sin ^{2} z+\frac{3(A-1)}{2 A}(\cos y \cos z-\cos x \sin y \sin z)^{2} \\
& -(A-1) X Z \frac{\sin (2 y)}{2 \sin x}-Y Z\left(A \cos ^{2} y+\sin ^{2} y\right) \frac{\cos x}{\sin ^{2} x} \\
& +\frac{Z^{2}}{2 \sin ^{2} x}\left(A \cos ^{2} y+\sin ^{2} y\right)+\frac{1}{2} X^{2}\left(A \sin ^{2} y+\cos ^{2} y\right)-Z
\end{aligned}
$$

where $A=a / b, C=c / b$ are positive dimensionless parameters with the restrictions

$$
C+1 \geq A, \quad A+1 \geq C, \quad A+C \geq 1
$$




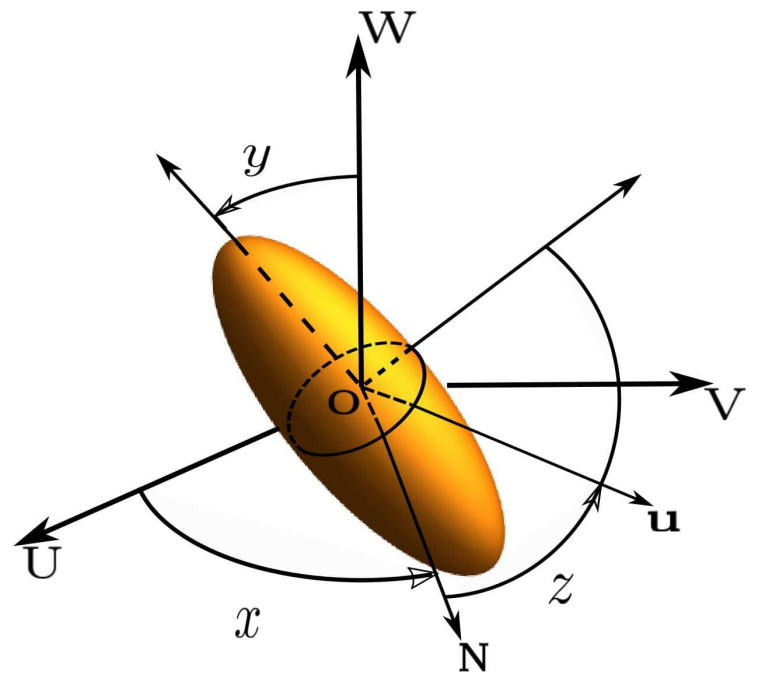

Fig. 1 Representation of the satellite in its coordinate frames: the orbital system $\mathrm{O} U V W$ and the system Ouvw moving with the satellite. The $\mathrm{O} N$ axis corresponds to the line of the nodes. The angles $x, y, z$ are the Euler angles.

This problem possesses 24 equilibria (see [17] and [19] for more details):

$$
\begin{array}{ll}
P_{1}=\left(\frac{\pi}{2}, \frac{\pi}{2}, 0,0,0,1\right), & P_{2}=\left(\frac{\pi}{2}, \frac{\pi}{2}, \frac{\pi}{2}, 0,0,1\right), \\
P_{3}=\left(\frac{\pi}{2}, 0,0,0,0, \frac{1}{A}\right), & P_{4}=\left(\frac{\pi}{2}, 0, \frac{\pi}{2}, 0,0, \frac{1}{A}\right), \\
P_{5}=\left(\frac{\pi}{2}, \frac{\pi}{2}, \pi, 0,0,1\right), & P_{6}=\left(-\frac{\pi}{2}, \frac{\pi}{2}, 0,0,0,1\right), \\
P_{7}=\left(-\frac{\pi}{2}, \frac{\pi}{2}, \pi, 0,0,1\right), & P_{8}=\left(\frac{\pi}{2},-\frac{\pi}{2}, 0,0,0,1\right), \\
P_{9}=\left(\frac{\pi}{2},-\frac{\pi}{2}, \pi, 0,0,1\right), & P_{10}=\left(-\frac{\pi}{2},-\frac{\pi}{2}, 0,0,0,1\right), \\
P_{11}=\left(-\frac{\pi}{2},-\frac{\pi}{2}, \pi, 0,0,1\right), & P_{12}=\left(\frac{\pi}{2}, \frac{\pi}{2},-\frac{\pi}{2}, 0,0,1\right), \\
P_{13}=\left(\frac{\pi}{2},-\frac{\pi}{2}, \frac{\pi}{2}, 0,0,1\right), & P_{14}=\left(\frac{\pi}{2},-\frac{\pi}{2},-\frac{\pi}{2}, 0,0,1\right), \\
P_{15}=\left(-\frac{\pi}{2}, \frac{\pi}{2}, \frac{\pi}{2}, 0,0,1\right), & P_{16}=\left(-\frac{\pi}{2}, \frac{\pi}{2},-\frac{\pi}{2}, 0,0,1\right), \\
P_{17}=\left(-\frac{\pi}{2},-\frac{\pi}{2}, \frac{\pi}{2}, 0,0,1\right), & P_{18}=\left(-\frac{\pi}{2},-\frac{\pi}{2},-\frac{\pi}{2}, 0,0,1\right), \\
P_{19}=\left(\frac{\pi}{2}, 0, \pi, 0,0, \frac{1}{A}\right), & P_{20}=\left(-\frac{\pi}{2}, 0,0,0,0, \frac{1}{A}\right), \\
P_{21}=\left(-\frac{\pi}{2}, 0, \pi, 0,0, \frac{1}{A}\right), & P_{22}=\left(\frac{\pi}{2}, 0,-\frac{\pi}{2}, 0,0, \frac{1}{A}\right), \\
P_{23}=\left(-\frac{\pi}{2}, 0, \frac{\pi}{2}, 0,0, \frac{1}{A}\right), & P_{24}=\left(-\frac{\pi}{2}, 0,-\frac{\pi}{2}, 0,0, \frac{1}{A}\right) .
\end{array}
$$

Due to the four independent discrete symmetries of the problem, given by

$$
\begin{aligned}
& S_{1}:(x, y, z, X, Y, Z) \longrightarrow(-x, y, z,-X, Y, Z), \\
& S_{2}:(x, y, z, X, Y, Z) \longrightarrow(x,-y,-z,-X, Y, Z), \\
& S_{3}:(x, y, z, X, Y, Z) \longrightarrow(x, y+\pi, z, X, Y, Z), \\
& S_{4}:(x, y, z, X, Y, Z) \longrightarrow(x, y, z+\pi, X, Y, Z),
\end{aligned}
$$


the study of their stability can be reduced to the analysis of the equilibria $P_{1}$, $P_{2}, P_{3}$ and $P_{4}$. The equivalence classes determined by the symmetries appear in Table 1 .

\begin{tabular}{|c|c|c|c|c|c|c|}
\hline$\left\{\begin{array}{c}P_{1} \\
P_{5} \\
\vdots \\
P_{11}\end{array}\right.$ & $S_{4}$ & $\left\{\begin{array}{l}P_{1} \\
P_{6} \\
P_{8} \\
P_{10}\end{array}\right.$ & $S_{1}$ & $\left\{\begin{array}{l}P_{1} \\
P_{8}\end{array}\right.$ & $S_{2}$ & $P_{1}$ \\
\hline$\left\{\begin{array}{c}P_{2} \\
P_{12} \\
\vdots \\
P_{18}\end{array}\right.$ & $S_{4}$ & $\left\{\begin{array}{l}P_{2} \\
P_{13} \\
P_{15} \\
P_{17}\end{array}\right.$ & $S_{1}$ & $\left\{\begin{array}{l}P_{2} \\
P_{13}\end{array}\right.$ & $S_{3}$ & $P_{2}$ \\
\hline$\left\{\begin{array}{c}P_{3} \\
P_{19} \\
P_{20} \\
P_{21}\end{array}\right.$ & $S_{4}$ & $\left\{\begin{array}{c}P_{3} \\
P_{20}\end{array}\right.$ & $S_{1}$ & $P_{3}$ & & \\
\hline$\left\{\begin{array}{c}P_{4} \\
P_{22} \\
P_{23} \\
P_{24}\end{array}\right.$ & $S_{4}$ & $\left\{\begin{array}{c}P_{4} \\
P_{23}\end{array}\right.$ & $S_{1}$ & $P_{4}$ & & \\
\hline
\end{tabular}

Table 1 The equivalence classes of equilibrium points $P_{j}$, for $j=1, \ldots, 24$ under the symmetries $S_{j}, j=1, \ldots, 4$.

In order to determine the stability regions of $P_{j}$, we introduce a linear symplectic change of variables $T_{j}$ associated to each point $P_{j}, j=1, \ldots, 4$, in the following way:

$$
\begin{aligned}
& T_{1}:(x, y, z, X, Y, Z) \longrightarrow\left(\bar{x}, \bar{y}+\frac{\pi}{2}, \bar{z}, \bar{X}, \bar{Y}, \bar{Z}+\frac{1}{A}-1\right), \\
& T_{2}:(x, y, z, X, Y, Z) \longrightarrow\left(\bar{x}, \bar{y}+\frac{\pi}{2}, \bar{z}+\frac{\pi}{2}, \bar{X}, \bar{Y}, \bar{Z}+\frac{1}{A}-1\right), \\
& T_{3}:(x, y, z, X, Y, Z) \longrightarrow(\bar{x}, \bar{y}, \bar{z}, \bar{X}, \bar{Y}, \bar{Z}), \\
& T_{4}:(x, y, z, X, Y, Z) \longrightarrow\left(\bar{x}, \bar{y}, \bar{z}+\frac{\pi}{2}, \bar{X}, \bar{Y}, \bar{Z}\right) .
\end{aligned}
$$

Then, we apply $T_{j}$ to Hamiltonian (1) and translate the equilibrium solution $P_{j}=\left(P_{j 1}, P_{j 2}, P_{j 3}, P_{j 4}, P_{j 5}, P_{j 6}\right)$ to the origin by means of the linear change of coordinates given by

$$
\begin{aligned}
& \bar{x}=x_{1}+P_{j 1}, \quad \bar{y}=y_{1}+P_{j 2}, \quad \bar{z}=z_{1}+P_{j 3}, \\
& \bar{X}=X_{1}+P_{j 4}, \quad \bar{Y}=Y_{1}+P_{j 5}, \quad \bar{Z}=Z_{1}+P_{j 6},
\end{aligned}
$$


with $j=1, \ldots, 4$. After these transformations are performed, the Hamiltonian function (1) assumes the same form in all cases, namely, the function

$$
\begin{aligned}
H= & \frac{1}{2} Y_{1}^{2}\left(\frac{A}{C}+\tan ^{2} x_{1}\left(A \cos ^{2} y_{1}+\sin ^{2} y_{1}\right)\right) \\
& +\frac{3(C-1)}{2 A} \cos ^{2} x_{1} \sin ^{2} z_{1} \\
& +\frac{3(A-1)}{2 A}\left(\sin x_{1} \sin y_{1} \sin z_{1}+\cos y_{1} \cos z_{1}\right)^{2} \\
& -\frac{1}{2}(A-1) X_{1} Y_{1} \tan x_{1} \sin \left(2 y_{1}\right)+\frac{1}{2} X_{1}^{2}\left(A \sin ^{2} y_{1}+\cos ^{2} y_{1}\right) \\
& -\frac{1}{2}(A-1) X_{1}\left(\frac{1}{A}+Z_{1}\right) \sec x_{1} \sin \left(2 y_{1}\right) \\
& +Y_{1}\left(\frac{1}{A}+Z_{1}\right) \tan x_{1} \sec x_{1}\left(A \cos ^{2} y_{1}+\sin ^{2} y_{1}\right) \\
& +\frac{1}{2}\left(\frac{1}{A}+Z_{1}\right)^{2} \sec { }^{2} x_{1}\left(A \cos ^{2} y_{1}+\sin ^{2} y_{1}\right)-\left(\frac{1}{A}+Z_{1}\right) .
\end{aligned}
$$

Proposition 1 The stability of the equilibria $P_{j}$ of Hamiltonian (1) is the same for all $j=1, \ldots, 24$.

Proof. By applying the symmetries $S_{j}$ and the transformations $T_{j}$, with $j=1, \ldots, 4$, as described above, we obtain the same Hamiltonian for the study of the stability of every $P_{j}$ with $j=1, \ldots, 24$.

Then, from now on we generically allude to the stability of the equilibrium $P$ for referring to any equilibrium $P_{j}$, with $j=1, \ldots, 24$.

Hamiltonian function (3) is expanded in Taylor series around $\mathbf{0}$, constant terms are dropped and we get an expansion of the form

$$
H=H_{2}+H_{3}+H_{4}+\cdots,
$$

where

$$
\begin{aligned}
H_{2}= & \frac{1}{2 A} x_{1}^{2}-\frac{(A-1)(3 A+1)}{2 A^{2}} y_{1}^{2}+\frac{3(C-A)}{2 A} z_{1}^{2}+\frac{1}{2} X_{1}^{2}+\frac{A}{2 C} Y_{1}^{2}+\frac{A}{2} Z_{1}^{2} \\
& +x_{1} Y_{1}-\frac{A-1}{A} X_{1} y_{1}, \\
H_{3}= & x_{1}^{2} Z_{1}-\frac{A-1}{A} y_{1}^{2} Z_{1}+\frac{3(A-1)}{A} x_{1} y_{1} z_{1}+A x_{1} Y_{1} Z_{1}-(A-1) X_{1} y_{1} Z_{1}, \\
H_{4}= & \frac{1}{3 A} x_{1}^{4}+\frac{(A-1)(3 A+1)}{6 A^{2}} y_{1}^{4}-\frac{C-A}{2 A} z_{1}^{4}+\frac{5}{6} x_{1}^{3} Y_{1}+\frac{2(A-1)}{3 A} y_{1}^{3} X_{1} \\
& -\frac{A-1}{2 A^{2}} x_{1}^{2} y_{1}^{2}-\frac{3(C-1)}{2 A} x_{1}^{2} z_{1}^{2}+\frac{3(A-1)}{2 A} y_{1}^{2} z_{1}^{2}+\frac{A}{2} x_{1}^{2} Y_{1}^{2}+\frac{A}{2} x_{1}^{2} Z_{1}^{2} \\
& +\frac{1}{2}(A-1) y_{1}^{2} X_{1}^{2}-\frac{1}{2}(A-1) y_{1}^{2} Z_{1}^{2}-\frac{A-1}{2 A} x_{1}^{2} y_{1} X_{1}-\frac{A-1}{A} y_{1}^{2} x_{1} Y_{1} \\
& -(A-1) x_{1} y_{1} X_{1} Y_{1} .
\end{aligned}
$$


The linearisation matrix associated to the equilibrium $P$ is

$$
B=\left(\begin{array}{cccccc}
0 & \frac{1-A}{A} & 0 & 1 & 0 & 0 \\
1 & 0 & 0 & 0 & \frac{A}{C} & 0 \\
0 & 0 & 0 & 0 & 0 & A \\
-\frac{1}{A} & 0 & 0 & 0 & -1 & 0 \\
0 & \frac{(A-1)(3 A+1)}{A^{2}} & 0 & \frac{A-1}{A} & 0 & 0 \\
0 & 0 & \frac{3(1-C)}{A} & 0 & 0 & 0
\end{array}\right)
$$

whose characteristic polynomial is $p_{B}(r)=a_{0}+a_{2} r^{2}+a_{4} r^{4}+r^{6}$ with

$$
\begin{aligned}
& a_{0}=\frac{12}{A C}(A-1)(C-1)(C-A), \\
& a_{2}=\frac{1}{A C}\left(9 A^{3}-15 A^{2} C-6 A^{2}+6 A C^{2}+13 A C-7 A-3 C^{2}-C+4\right), \\
& a_{4}=\frac{1}{A C}\left(-3 A^{2} C-3 A^{2}+3 A C^{2}+2 A C+2 A-C+1\right) .
\end{aligned}
$$

We write $p_{B}(t)=a_{0}+a_{2} t+a_{4} t^{2}+t^{3}$ with $t=r^{2}$ and apply Sturm's theorem to determine the conditions on the parameters to obtain three distinct nonpositive real roots of the cubic polynomial $p_{B}(t)$. These values will correspond with six pure imaginary or zero roots of the polynomial $p_{B}(r)$. The polynomials in the Sturm sequence are

$$
\begin{aligned}
& p_{0}(t)=p_{B}(t), \\
& p_{1}(t)=a_{2}+2 a_{4} t+3 t^{2}, \\
& p_{2}(t)=b_{0}+b_{1} t, \\
& p_{3}(t)=\frac{9 \alpha_{1} \alpha_{2}^{2}}{4 \alpha_{3}^{2}},
\end{aligned}
$$

where $b_{j}$ and $\alpha_{j}$ are given in Table 5 of Appendix 8. By calculating the limit when $t \rightarrow-\infty$ of each sequence's term we get the following sequence of signs, $\left\{-,+,-\operatorname{sign}\left(b_{1}\right), \operatorname{sign}\left(\alpha_{1}\right)\right\}$. Let us denote the number of sign changes in this sequence by $V(-\infty)$. The evaluation of the sequence at 0 results in $\left\{\operatorname{sign}\left(a_{0}\right)\right.$, $\left.\operatorname{sign}\left(a_{2}\right), \operatorname{sign}\left(b_{0}\right), \operatorname{sign}\left(\alpha_{1}\right)\right\}$. The number of sign changes in this sequence is denoted by $V(0)$. Then, the number of distinct non-positive real roots of $p_{B}(t)$ is $V(-\infty)-V(0)$. For this number to be three, the following conditions must be fulfilled: $a_{0}>0, a_{2}>0, b_{0}>0, b_{1}>0, \alpha_{1}>0$. These requirements are satisfied in region $I$, that is the finite region enclosed by the straight lines $A=C, C=1$ and $A=1-C$, and in region II, that is the finite region enclosed by the straight lines $A=C, A=1, A=C-1$ and the curve $\alpha_{1}=0$, that comes from the last term of the Sturm sequence, see Fig. 2. These are the regions found by Beletskii [3,4] and Delp and DeBra [11], [10]. 


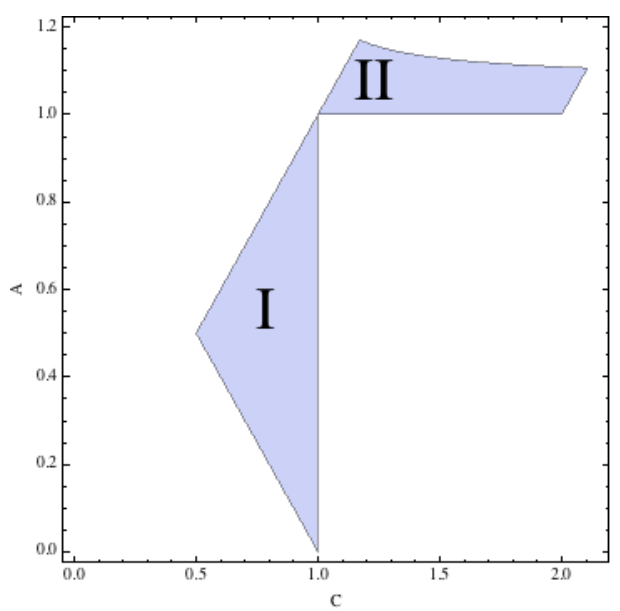

Fig. 2 Regions in the parameter plane defined by $A$ and $C$ where the necessary conditions for the equilibrium point to be linearly stable are satisfied.

The eigenvalues of matrix $B$ are $\lambda_{1}= \pm i \omega_{1}, \lambda_{2}= \pm i \omega_{2}, \lambda_{3}= \pm i \omega_{3}$, whose frequencies read as

$$
\omega_{1}=\frac{\sqrt{\beta+\sqrt{\alpha_{1}}}}{\sqrt{2 A C}}, \quad \omega_{2}=\frac{\sqrt{\beta-\sqrt{\alpha_{1}}}}{\sqrt{2 A C}}, \quad \omega_{3}=\sqrt{3(C-A)},
$$

with $\alpha_{1}$ comes from the last term in the Sturm sequence, that we repeat for the sake of clarity:

$\alpha_{1}=9 A^{4}-12 A^{3}(C+1)-2 A^{2}\left(6 C^{2}-15 C+1\right)+4 A\left(3 C^{2}-4 C+1\right)+(C-1)^{2}$

and

$$
\beta=A(-3 A+2 C+2)-C+1 .
$$

The frequencies are $\omega_{1}>\omega_{2}>0, \omega_{3}>0$ and thence, the eigenvalues are pure imaginary in regions I and II and on the boundaries $A=1-C$ with $C \in(1 / 2,1)$; and $A=C-1$ with $A \in(1,(-3+8 \sqrt{6}) / 15]$. In all these cases the equilibrium is elliptic, matrix $B$ is diagonalisable and therefore, the point is linearly stable. On the rest of the boundary of regions I and II matrix $B$ is non-diagonalisable. Outside regions I and II the equilibrium is unstable.

For simplification purposes it is interesting to have the expressions of the fundamental parameters $A$ and $C$ as functions of the frequencies $\omega_{1}$ and $\omega_{2}$, as well as $\omega_{3}\left(\omega_{1}, \omega_{2}\right)$, at hand:

$$
\begin{gathered}
A=-\frac{\left(\omega_{1}^{2}-4\right)\left(\omega_{2}^{2}-4\right)}{3\left(\omega_{1}^{2} \omega_{2}^{2}-4\right)}, \quad C=-\frac{16\left(\omega_{1}^{2}-1\right)\left(\omega_{2}^{2}-1\right)}{\left(\omega_{1}^{2} \omega_{2}^{2}-4\right)\left(\omega_{1}^{2} \omega_{2}^{2}+4\left(1-\omega_{1}^{2}-\omega_{2}^{2}\right)\right)}, \\
\omega_{3}^{2}=\frac{16\left(\omega_{1}^{2}-1\right)^{2}-4\left(2 \omega_{1}^{4}-\omega_{1}^{2}+8\right) \omega_{2}^{2}+\left(\omega_{1}^{2}-4\right)^{2} \omega_{2}^{4}}{\left(\omega_{1}^{2} \omega_{2}^{2}-4\right)\left(4-4 \omega_{2}^{2}+\omega_{1}^{2}\left(\omega_{2}^{2}-4\right)\right)} .
\end{gathered}
$$


Now we introduce a real linear symplectic change of coordinates to put $\mathrm{H}_{2}$ in linear normal form by using the eigenvalues and eigenvectors of matrix $B$. We call $\mathbf{x}=\left(q_{1}, q_{2}, q_{3}, p_{1}, p_{2}, p_{3}\right)$ the transformed variables, where $q_{i}$ stand for coordinates and $p_{i}$ do for their conjugate momenta. Using Markeev's procedure for the normalisation of the quadratic terms in rectangular coordinates, see details in [22], $\mathrm{H}_{2}$ is converted into

$$
H_{2}(\mathbf{I})=\omega_{1} I_{1}+\omega_{2} I_{2}+\omega_{3} I_{3},
$$

in region $\mathrm{I}$ and

$$
H_{2}(\mathbf{I})=\omega_{1} I_{1}-\omega_{2} I_{2}+\omega_{3} I_{3},
$$

in region II, where $(\mathbf{I}, \theta)=\left(I_{1}, I_{2}, I_{3}, \theta_{1}, \theta_{2}, \theta_{3}\right)$ are the usual action-angle variables, with $I_{j}=\frac{1}{2}\left(q_{j}^{2}+p_{j}^{2}\right)$ the actions conjugate to the angles $\theta_{j}=$ $\tan ^{-1}\left(p_{j} / q_{j}\right)$ for $j=1,2,3$. Thus, the equilibrium is nonlinearly (Liapunov) stable in region I by Dirichlet's Theorem [13], and a nonlinear stability analysis is required in region II, as found by Beletskii [4]. That is why our study will be focused in region II from now on. The linear symplectic change from $\left(x_{1}, y_{1}, z_{1}, X_{1}, Y_{1}, Z_{1}\right)$ to $\mathbf{x}$ can be provided by the first author.

We continue with the computation of the normal form of Hamiltonian $H$ appearing in (4). It is convenient to introduce a complex linear change to express $H_{2}$ in complex diagonal form. The two changes applied to $H_{2}$ up to now are also applied to the higher-order terms. Subsequently, a standard procedure based on Lie transformations $[12,25]$ is performed to normalise the terms from $\mathrm{H}_{3}$ on, so that the resulting Hamiltonian commutes with $\mathrm{H}_{2}$ at every step. This process is executed up to a finite order and, in most of the cases in this paper, order two is enough, which means including the polynomials of degree four in $\mathbf{x}$ that define $H_{4}$.

We denote by $\mathcal{H}_{p}$ the normalisation of $H_{p}$, the terms composed by homogeneous polynomials of degree $p$ in $\mathbf{x}$, and by $\mathcal{H}^{p}$ the normal form truncated at order $p-2$. Thus,

$$
\mathcal{H}^{p}(\mathbf{x})=H_{2}(\mathbf{x})+\mathcal{H}_{3}(\mathbf{x})+\cdots+\mathcal{H}_{p}(\mathbf{x})
$$

represents the truncation of the normal form at terms of degree $p$ in rectangular coordinates. The Poisson brackets $\left\{H_{2}, \mathcal{H}_{k}\right\}=0$ for $k=3, \ldots, p$. The normal form is achieved through the calculation of the associated generating function, which is used to define the symplectic transformation.

The normalised Hamiltonian put in action-angle variables corresponding to $(7)$ reads as

$$
\mathcal{H}^{p}(\mathbf{I}, \theta)=H_{2}(\mathbf{I})+\mathcal{H}_{3}(\mathbf{I}, \theta)+\cdots+\mathcal{H}_{p}(\mathbf{I}, \theta),
$$

where the dependence of $\mathcal{H}_{j}$ with respect to $\theta$ occurs only due to the possible resonances of $H_{2}$. In fact, Hamiltonians $\mathcal{H}_{j}(\mathbf{I}, \theta)$ can be rewritten as $\mathcal{H}_{j}(\mathbf{I}, \phi)$ where $\phi=\left(\phi_{1}, \ldots, \phi_{s}\right), \phi_{i}=\mathbf{k}^{i} \cdot \theta$, with the vectors $\mathbf{k}^{i}$ introduced in Appendix 7 . The operator $(\cdot)$ stands for the usual inner product of two vectors. It is stressed that $\mathcal{H}_{j}$ may be independent of some angles $\phi_{j}$ or even of all of them. 
Remark 1 Using action-angle variables defined above, Hamiltonian function $\mathcal{H}^{p}$ in (7) leads to a finite Fourier series in $\theta$ whose coefficients are polynomials in $\sqrt{I_{i}}$, with terms of the form

$c I_{1}^{\alpha_{1} / 2} \cdots I_{n}^{\alpha_{n} / 2} \cos \left(\beta_{1} \theta_{1}+\cdots+\beta_{n} \theta_{n}\right), \quad s I_{1}^{\alpha_{1} / 2} \cdots I_{n}^{\alpha_{n} / 2} \sin \left(\beta_{1} \theta_{1}+\cdots+\beta_{n} \theta_{n}\right)$,

where $c, s$ are real constants, the $\alpha_{j}$ are non-negative integers and the $\beta_{j}$ are integers. Since the Fourier series came from a real power series the terms above satisfy the so called d'Alembert character [25], i.e., the following relations hold:

$$
\text { for } j=1, \ldots, n, \quad \alpha_{j} \geq\left|\beta_{j}\right| \quad \text { and } \quad \alpha_{j} \equiv \beta_{j} \quad \bmod 2 .
$$

We observe that $H$ in (8) is an analytic function of the variables $\sqrt{I_{j}}, \theta_{j}$ and is $2 \pi$-periodic in $\theta_{j}, j=1, \ldots, n$ excepting at $\mathbf{I}=\mathbf{0}$. To circumvent the problem at the origin of $\mathbb{R}^{6}$ one proceeds by keeping track of the d'Alembert character of the Hamiltonians and related formulae in action-angle coordinates. If this character is maintained through the different manipulations, transforming these formulae back to rectangular coordinates, the resulting expressions are polynomials in $\mathbf{x}$, thus analytic everywhere. Throughout the text we have checked that all Hamiltonians and related functions satisfy (9), hence all the calculations are performed in the analytic setting.

Returning to the normal form Hamiltonian (4), when no resonance of order three and four are present in the normal form Hamiltonian we write

$$
H(\mathbf{I}, \theta)=H_{2}(\mathbf{I})+\mathcal{H}_{4}(\mathbf{I})+\cdots,
$$

where

$$
\mathcal{H}_{4}(\mathbf{I})=c_{200} I_{1}^{2}+c_{020} I_{2}^{2}+c_{002} I_{3}^{2}+c_{110} I_{1} I_{2}+c_{011} I_{2} I_{3}+c_{101} I_{1} I_{3},
$$

and

$$
\begin{aligned}
c_{200}= & \frac{f_{2}}{192 f_{10} g_{1} h_{1}^{2} h_{2}}\left(4\left(-4 g_{1}^{3} g_{2}-4 g_{1}^{2} g_{3} \omega_{1}^{2}+g_{6} \omega_{1}^{4}+g_{5} \omega_{1}^{6}+g_{2} g_{4} \omega_{1}^{8} \omega_{2}^{2}\right)\right. \\
& \left.-h_{2}\left(4 g_{1}^{2} g_{7}+g_{9} \omega_{1}^{2}+g_{2} g_{8} \omega_{1}^{4}\right) \omega_{3}^{2}\right) \\
c_{020}= & \frac{g_{2}}{192 g_{10} f_{1} h_{1}^{2} h_{2}}\left(4\left(-4 f_{1}^{3} f_{2}-4 f_{1}^{2} f_{3} \omega_{2}^{2}+f_{6} \omega_{2}^{4}+f_{5} \omega_{2}^{6}+f_{2} f_{4} \omega_{1}^{2} \omega_{2}^{8}\right)\right. \\
& \left.-h_{2}\left(4 f_{1}^{2} f_{7}+f_{9} \omega_{2}^{2}+f_{2} f_{8} \omega_{2}^{4}\right) \omega_{3}^{2}\right), \\
c_{002}= & -\frac{A}{4}, \\
c_{110}= & \frac{8 t_{4} \omega_{1}^{2}+2 t_{3} \omega_{1}^{4}-2 t_{2} \omega_{1}^{6}+t_{1} \omega_{1}^{8}-128 g_{1} t_{19} \omega_{2}^{2}-2 t_{5} \omega_{1}^{10} \omega_{2}^{2}+t_{18} \omega_{1}^{12} \omega_{2}^{2}}{48 h_{1}^{2} h_{2} h_{3} \omega_{1} \omega_{2}} \\
c_{011}= & \frac{64 g_{1} t_{17}-16 t_{16} \omega_{1}^{2}+4 t_{14} \omega_{1}^{4}+t_{15} \omega_{1}^{6}+g_{2} t_{13} \omega_{1}^{8}+g_{2}^{2} t_{12} \omega_{1}^{10}}{24 g_{10} h_{1} h_{2} h_{3} \omega_{2}\left(4+g_{2} \omega_{1}^{2}-4 \omega_{2}^{2}\right) \omega_{3}} \\
c_{101}= & \frac{1}{24 f_{10} h_{1} h_{2} h_{3} \omega_{1}\left(4+g_{2} \omega_{1}^{2}-4 \omega_{2}^{2}\right) \omega_{3}}\left(-16 g_{1} t_{11} \omega_{1}^{2}+4 t_{8} \omega_{1}^{4}-t_{6} \omega_{1}^{6}\right. \\
& \left.+t_{7} \omega_{1}^{8}-g_{2} t_{9} \omega_{1}^{10}+g_{2} t_{10} \omega_{1}^{12}+g_{2}^{3} t_{20} \omega_{1}^{14}-256 g_{1}^{2} t_{21} \omega_{3}^{2}\right),
\end{aligned}
$$


with $f_{j}, g_{j}, h_{j}, t_{j}$ functions of $\omega_{i}$ that are given in Tables 6 and 7 of Appendix 8 . Note that in this generic normal form Hamiltonian odd terms $\mathcal{H}_{3}, \mathcal{H}_{5}, \ldots$ are always zero. The expressions of the corresponding two generating transformation's terms are lengthy, thus we do not include them in the paper, but they are available upon request from the first author. In particular, the generating function associated to the normal form Hamiltonian is needed to build the transformation explicitly.

The reader should be aware that this normal form is valid in the absence of certain resonances, as we explain below.

The normal form truncated at polynomials of degree four in rectangular coordinates (that is, at order two of the Lie transformation) is meaningful when there are no resonances of orders three and four. Taking into account Definition 1 in Apppendix 7, there are exactly 21 possible distinct resonance vectors of orders three and four, namely,

$$
\begin{array}{llll}
\mathbf{k}^{1}=(0,1,2), & \mathbf{k}^{2}=(0,2,1), & \mathbf{k}^{3}=(1,2,0), & \mathbf{k}^{4}=(1,-1,-1), \\
\mathbf{k}^{5}=(1,0,-2), & \mathbf{k}^{6}=(1,1,-1), & \mathbf{k}^{7}=(2,0,-1), & \mathbf{k}^{8}=(0,1,3), \\
\mathbf{k}^{9}=(0,3,1), & \mathbf{k}^{10}=(1,2,1), & \mathbf{k}^{11}=(1,3,0), & \mathbf{k}^{12}=(0,2,2), \\
\mathbf{k}^{13}=(1,-2,-1), & \mathbf{k}^{14}=(1,-1,-2), & \mathbf{k}^{15}=(1,0,-3), & \mathbf{k}^{16}=(1,1,-2), \\
\mathbf{k}^{17}=(1,2,-1), & \mathbf{k}^{18}=(2,-1,-1), & \mathbf{k}^{19}=(3,0,-1), & \mathbf{k}^{20}=(2,1,-1), \\
\mathbf{k}^{21}=(2,0,-2) & &
\end{array}
$$

After the analysis of the coefficient's denominators of the function $\mathcal{H}_{4}(\mathbf{I})$ and the corresponding generating functions of first and second order needed to determine $\mathcal{H}_{4}$ (see details on the normalisation procedure based on Lie transformations in [12]).

Taking into account that $\omega_{1}>\omega_{2}>0, \omega_{3}>0$ and $(C, A) \in$ region II, we arrive at the fact that there are nine cases where the normalised Hamiltonian (11) does not apply. These are the resonances satisfying $\mathbf{k}^{i} \cdot \omega=0$ for $i=$ $2,4,6,7,11,12,14,16,21$, see Fig. 3 . Here and from now on $\omega=\left(\omega_{1},-\omega_{2}, \omega_{3}\right)$. In each of these cases, a specific normal form has been calculated. However for the remaining 12 resonances the normal form transformation determined above works properly because in these cases the resonant part of the Hamiltonian normal form is null. Then, in these twelve resonances the normalised Hamiltonian including terms of order four has the form (10). For a specific point in the plane of parameters $(C, A)$ which does not belong to a resonance curve but is near to it, the normal form can produce the appearance of small divisors in case of non-Diophantine frequencies $\sigma_{i}$ introduced in (23), but one can avoid them, as it is explained in Remark 7 of Sect. 3. 


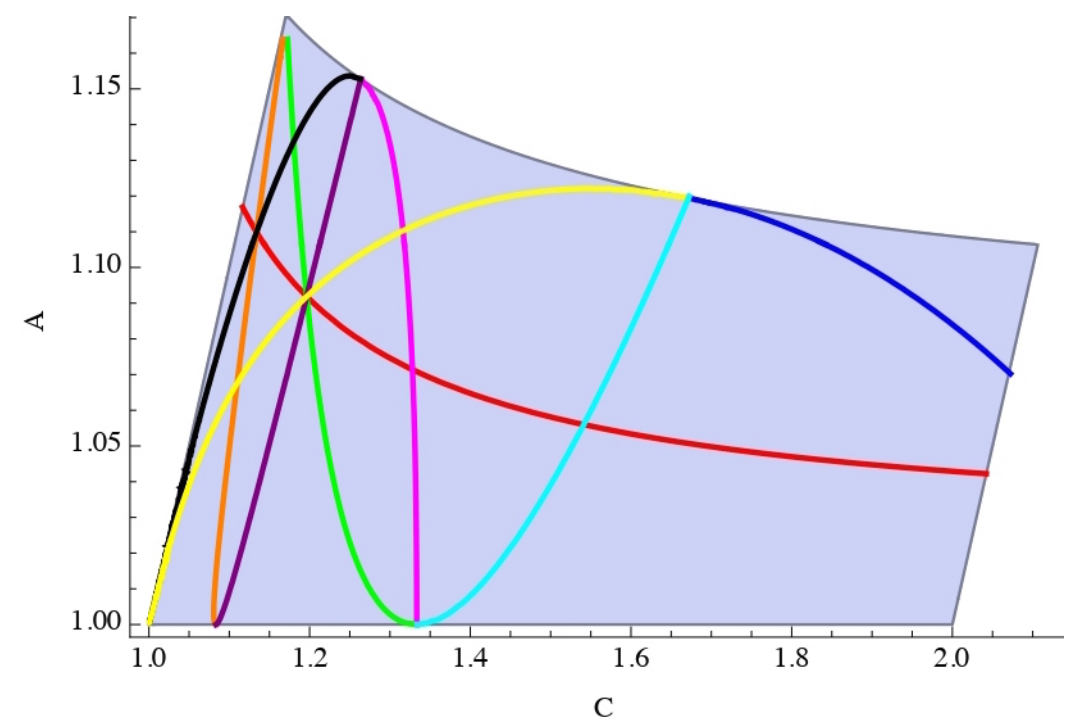

Fig. 3 Representation in region II of the nine curves as functions of the parameters $(C, A)$ where the normal form (10) truncated at order 2 is not well defined. They correspond to the following single resonances: $\mathbf{k}^{2}:-2 \omega_{2}=\omega_{3}, \quad \mathbf{k}^{4}:-\omega_{1}+\omega_{2}=\omega_{3}, \quad \mathbf{k}^{6}:-\omega_{1}=$ $\omega_{2}+\omega_{3}, \mathbf{k}^{7}:-2 \omega_{1}=\omega_{3}, \quad \mathbf{k}^{11}:-\omega_{1}=3 \omega_{2}, \quad \mathbf{k}^{12}:-\omega_{2}=\omega_{3}, \quad \mathbf{k}^{14}:-\omega_{1}+\omega_{2}=$ $2 \omega_{3}, \quad \mathbf{k}^{16}:-\omega_{1}=\omega_{2}+2 \omega_{3}, \quad \mathbf{k}^{21}:-\omega_{1}=\omega_{3}$.

\section{Lie stability of the equilibrium point}

We plan to determine the Lie stability regions in II, see Definition 6 in Appendix 7 . To simplify the discussion, we consider $\bar{\omega}_{1}=\omega_{1} / \omega_{3}, \bar{\omega}_{2}=\omega_{2} / \omega_{3}$, $\bar{\omega}_{3}=1$. The first step is the determination of the set $S$ (see the definition in Appendix 7). This passes through the construction of the formal integrals $F_{i}$ associated to $H_{2}$. The number of linearly independent integrals is $1 \leq d(=3-s) \leq 3$. On the one hand, when $\mathbf{I} \in S$ then $H_{2}(\mathbf{I})=0$ and by applying (6) we can write

$$
I_{2}=\frac{1}{\bar{\omega}_{2}}\left(\bar{\omega}_{1} I_{1}+I_{3}\right)
$$

with $I_{1}, I_{3} \geq 0$. On the other hand,

$$
k_{1} \bar{\omega}_{1}-k_{2} \bar{\omega}_{2}+k_{3}=0
$$

with $\mathbf{k}=\left(k_{1}, k_{2}, k_{3}\right) \in \mathbb{Z}^{3}$. The following situations are in order:

$\left(a_{1}\right)$ If $\bar{\omega}_{1}, \bar{\omega}_{2} \in \mathbb{Q}$, then we obtain $F_{1}=\bar{\omega}_{1} I_{1}-\bar{\omega}_{2} I_{2}+I_{3}, d=1, s=2$ and

$$
S=\left\{\left(I_{1}, \frac{1}{\bar{\omega}_{2}}\left(\bar{\omega}_{1} I_{1}+I_{3}\right), I_{3}\right) \mid I_{1}, I_{3} \geq 0\right\} .
$$


$\left(a_{2}\right)$ If $\bar{\omega}_{1} \in \mathbb{Q}$ and $\bar{\omega}_{2} \in \mathbb{R} \backslash \mathbb{Q}$, then one gets $F_{1}=\bar{\omega}_{1} I_{1}+I_{3}, F_{2}=I_{2}$. Thus, $d=2, s=1$ and

$$
S=\left\{\left(I_{1}, I_{2}, I_{3}\right) \mid \bar{\omega}_{1} I_{1}+I_{3}=0, I_{2}=0, I_{1}, I_{3} \geq 0\right\}=\{\mathbf{0}\}
$$

$\left(b_{1}\right)$ If $\bar{\omega}_{1}, \bar{\omega}_{2} \in \mathbb{R} \backslash \mathbb{Q}$ and there is no integer vector $\mathbf{k}=\left(k_{1}, k_{2}, k_{3}\right) \neq(0,0,0)$ satisfying (13) we conclude that there are no resonances among the $I_{j}$. Hence, $F_{j}=I_{j}$ for $j=1,2,3$. Therefore, $d=3, s=0$ and $S=\{\mathbf{0}\}$.

$\left(b_{2}\right)$ If $\bar{\omega}_{1}, \bar{\omega}_{2} \in \mathbb{R} \backslash \mathbb{Q}$ and there is an integer vector $\mathbf{k} \neq \mathbf{0}$ satisfying (13), then

$$
\bar{\omega}_{1}=\frac{k_{2}}{k_{1}} \bar{\omega}_{2}-\frac{k_{3}}{k_{1}}
$$

with $k_{1} \neq 0$. Notice that $k_{1}=0$ would imply $-\bar{\omega}_{2} k_{2}+k_{3}=0$ and then, either $k_{2}=0$, in which case $k_{1}=k_{2}=k_{3}=0$ that is impossible, or $\bar{\omega}_{2}=k_{3} / k_{2} \in \mathbb{Q}$, contradicting the hypotheses of $\left(b_{2}\right)$. Analogously $k_{2} \neq 0$ because $k_{2}=0$ would lead to $\bar{\omega}_{1}=-k_{3} / k_{1} \in \mathbb{Q}$ that is not feasible. However, $k_{3}=0$ is possible and this implies $k_{1} \bar{\omega}_{1}=k_{2} \bar{\omega}_{2}$.

Using (14) we get

$$
H_{2}=\left(\frac{k_{2}}{k_{1}} I_{1}-I_{2}\right) \bar{\omega}_{2}-\frac{k_{3}}{k_{1}} I_{1}+I_{3}
$$

from where we deduce that $F_{1}=k_{2} I_{1} / k_{1}-I_{2}, F_{2}=-k_{3} I_{1} / k_{1}+I_{3}, d=2$ and $s=1$.

Consider the set

$$
K=\left\{\mathbf{k}=\left(k_{1}, k_{2}, k_{3}\right) \in \mathbb{Z}^{3} \mid k_{1} \neq 0, k_{2} / k_{1}>0, k_{3} / k_{1} \geq 0\right\}
$$

If $\mathbf{k} \in K$ then $\operatorname{dim} S=1$ with

$$
S=\left\{\left(I_{1}, \frac{k_{2}}{k_{1}} I_{1}, \frac{k_{3}}{k_{1}} I_{1}\right) \mid I_{1} \geq 0\right\} .
$$

If $\mathbf{k} \notin K$ from $F_{1}$ and $F_{2}$ it is readily deduced that $S=\{\mathbf{0}\}$.

$\left(b_{3}\right)$ If $\bar{\omega}_{1} \in \mathbb{R} \backslash \mathbb{Q}$ and $\bar{\omega}_{2} \in \mathbb{Q}$, then we take $F_{1}=I_{1}, F_{2}=-\bar{\omega}_{2} I_{2}+I_{3}$, from where we get $d=2, s=1$ and

$$
S=\left\{\left(0, \frac{1}{\bar{\omega}_{2}} I_{3}, I_{3}\right) \mid I_{3} \geq 0\right\} .
$$

Remark 2 According to Remark 12 in Appendix 7, when $S=\{\mathbf{0}\}$ Lie stability holds. Therefore in cases $\left(a_{2}\right),\left(b_{1}\right)$ and $\left(b_{2}\right)$ with $\mathbf{k} \notin K$, Lie stability is concluded directly from $\mathrm{H}_{2}$ regardless of the higher-order terms. 
In the remaining cases (i.e. Remark 2 does not hold) the analysis of the higher-order terms is necessary. In accordance to Theorem 4 we have to evaluate $\mathcal{H}^{4}$ in $S \backslash\{\mathbf{0}\}$ and determine the regions where it does not change sign. First, we consider the generic (non-resonant) situations and analyse the regions in II where Hamiltonian (11) evaluated in $S \backslash\{\mathbf{0}\}$ does not change sign.

Consider $\mathbf{I} \in S \backslash\{\mathbf{0}\}$ and replace (12) in (11) to get

$$
\mathcal{H}_{4}(\mathbf{I})=\beta_{1} I_{1}^{2}+\beta_{2} I_{1} I_{3}+\beta_{3} I_{3}^{2}
$$

with

$$
\begin{aligned}
& \beta_{1}=\frac{1}{\bar{\omega}_{2}^{2}}\left(c_{020} \bar{\omega}_{1}^{2}+c_{110} \bar{\omega}_{1} \bar{\omega}_{2}+c_{200} \bar{\omega}_{2}^{2}\right), \\
& \beta_{2}=\frac{1}{\bar{\omega}_{2}^{2}}\left(2 c_{020} \bar{\omega}_{1}+c_{110} \bar{\omega}_{2}+c_{011} \bar{\omega}_{1} \bar{\omega}_{2}+c_{101} \bar{\omega}_{2}^{2}\right), \\
& \beta_{3}=\frac{1}{\bar{\omega}_{2}^{2}}\left(c_{020}+c_{011} \bar{\omega}_{2}+c_{002} \bar{\omega}_{2}^{2}\right) .
\end{aligned}
$$

When $\mathcal{H}_{4}(\mathbf{I})=0$, then for $\beta_{1} \neq 0$,

$$
I_{1}=\frac{-\beta_{2} \pm \sqrt{\beta_{2}^{2}-4 \beta_{1} \beta_{3}}}{2 \beta_{1}} I_{3}
$$

which defines a line through the origin of the $I_{1} I_{3}$-plane. Taking into account that $I_{1}, I_{3} \geq 0$, if the line's slope is positive then $\mathcal{H}_{4}(\mathbf{I})=0$ for $I_{1}, I_{3}>0$, therefore $\mathcal{H}_{4}(\mathbf{I})$ changes sign for $I_{1}, I_{3}>0$. When $\beta_{1}=0$, then $I_{3}=0$ and $I_{1}=-\beta_{3} I_{3} / \beta_{2}$. Thus, $\mathcal{H}_{4}(\mathbf{I})$ changes sign in the regions where $\beta_{1} \neq 0$ and

$$
\frac{-\beta_{2}+\sqrt{\beta_{2}^{2}-4 \beta_{1} \beta_{3}}}{2 \beta_{1}}>0 \quad \text { or } \quad \frac{-\beta_{2}-\sqrt{\beta_{2}^{2}-4 \beta_{1} \beta_{3}}}{2 \beta_{1}}>0
$$

and in the regions where $\beta_{1}=0$ and $\beta_{3} / \beta_{2}<0$. If one of the slopes is positive and the other one is zero, one has that (15) also changes sign. In summary, Hamiltonian (15) does not change sign in the dark-blue region of Fig. 4, whereas the light-blue region stands for the values where the normal form (15) changes sign. 


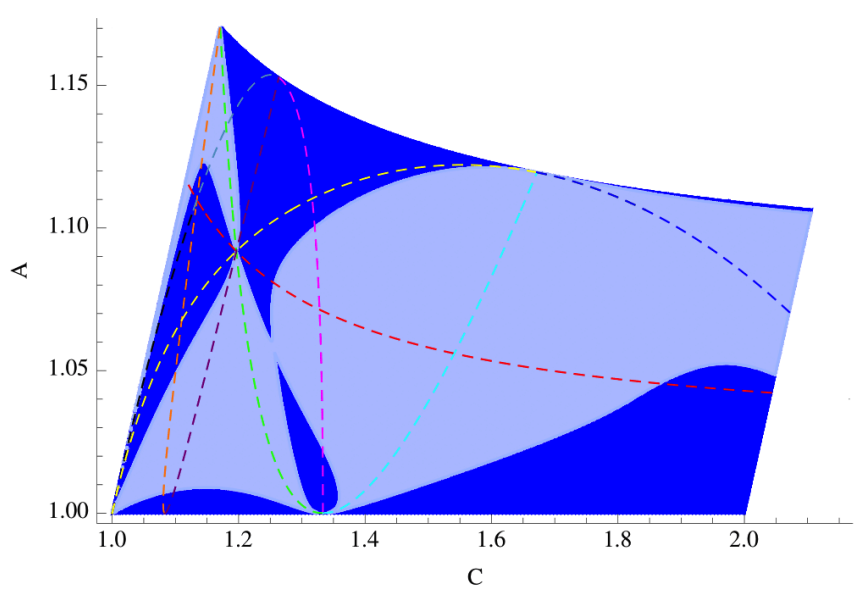

Fig. 4 Cases $\left(a_{1}\right): \bar{\omega}_{1}, \bar{\omega}_{2} \in \mathbb{Q}$ and $\left(b_{2}\right): \bar{\omega}_{1}, \bar{\omega}_{2} \in \mathbb{R} \backslash \mathbb{Q}$ with $\mathbf{k} \in K$. The dark-blue region corresponds to the Lie stable cases. In the light-blue region the normal form (15) changes sign. The coloured curves stand for the resonances appearing in Fig. 3, where (15) does not apply.

Consequently, when Hamiltonian $\mathrm{H}_{2}$ is not enough to conclude Lie stability, we need to consider different situations, accordingly with the analysis made in the above paragraphs. We proceed as follows:

$\left(a_{1}\right)$ By virtue of Theorem 4 the equilibrium $P$ is Lie stable whenever $(C, A)$ belongs to the dark-blue region.

$\left(b_{2}\right)$ Taking $\mathbf{I} \in S \backslash\{\mathbf{0}\}$ and replacing conveniently in (15) we get

$$
\mathcal{H}^{4}(\mathbf{I})=\left(\beta_{1}+\beta_{2} \frac{k_{3}}{k_{1}}+\beta_{3} \frac{k_{3}^{2}}{k_{1}^{2}}\right) I_{1}^{2},
$$

with $\beta_{1}, \beta_{2}, \beta_{3}$ given in (16). The analysis of the sign of $\mathcal{H}^{4}(\mathbf{I})$ coincides with the one performed above. Thence, using Theorem 4 the equilibrium $P$ is Lie stable whenever $(C, A)$ belongs to the dark-blue region.

$\left(b_{3}\right)$ Restricting $\mathbf{I}$ to be in $S \backslash\{\mathbf{0}\}$, we have that

$$
\mathcal{H}^{4}(\mathbf{I})=\beta_{3} I_{3}^{2},
$$

with $\beta_{3}$ given in (16). The coefficient $\beta_{3} \equiv \beta_{3}(C, A)$ is zero on the black lines appearing in Fig. 5. Therefore, the application of Theorem 4 allows us to conclude that the equilibrium $P$ is Lie stable in the whole region II, excepting on the black curves in Fig. 5 . When $\beta_{3}=0$ a higher-order normalisation would be required, but we do not pursue it in this paper. 


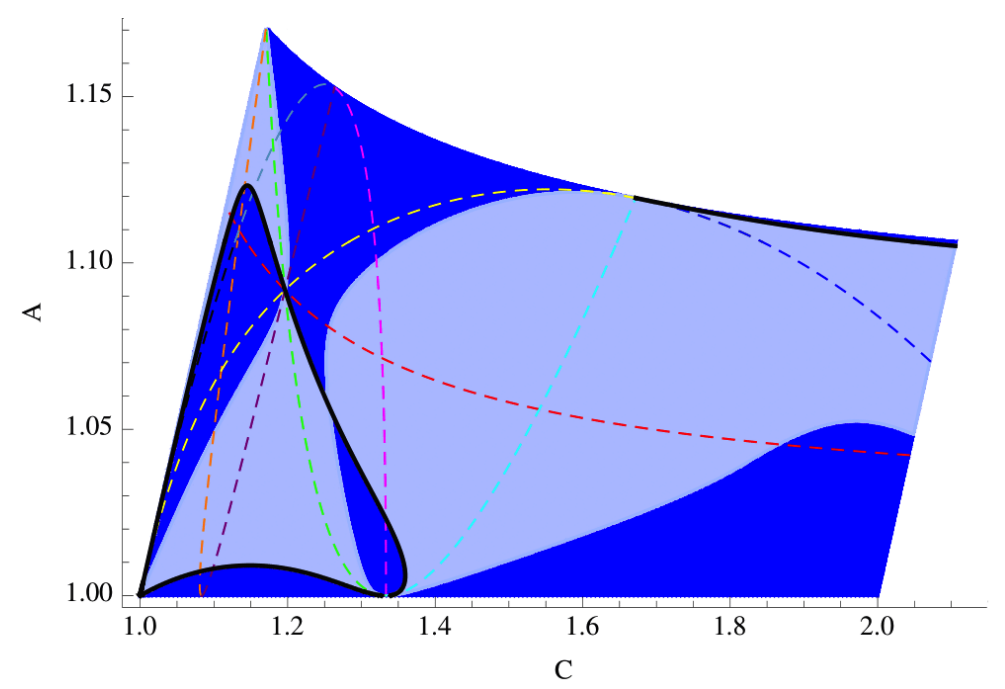

Fig. 5 Case $\left(b_{3}\right): \bar{\omega}_{1} \in \mathbb{R} \backslash \mathbb{Q}, \bar{\omega}_{2} \in \mathbb{Q}$. The light-blue and dark-blue regions correspond to the Lie stable cases. On the black curves the normal form is null. The coloured curves stand for the resonances appearing in Fig. 3, where (15) does not apply.

It is instructive to compare the analysis performed so far with the one applying Nekhoroshev stability theory accomplished in Appendix 9. We note that the curves separating the blue and the light-blue regions of Figs. 4 and 5 are the same as the curves separating the quasi-convex and directionally quasiconvex regions of Fig. 9. However we obtain more stable cases, in particular those of Remark 2 and the ones corresponding to case $\left(b_{3}\right)$ (see Fig. 5). See also point 6 in Sect. 6 .

Once the main regions in the parametric plane $(C, A)$ have been analysed we need to deal with the nine resonances appearing in Fig. 3. We classify them into four groups.

1. Resonances defined through $\mathbf{k}^{2}=(0,2,1)$, i.e. $2 \omega_{2}=\omega_{3}$ and $2 \bar{\omega}_{2}=1$; and $\mathbf{k}^{12}=(0,2,2)$, i.e. $\omega_{2}=\omega_{3}$ and $\bar{\omega}_{2}=1$.

These resonances belong either to case $\left(a_{1}\right)$ or to case $\left(b_{3}\right)$, therefore $\operatorname{dim} S=2$ or $\operatorname{dim} S=1$, respectively.

- For $\mathbf{k}^{2}$ the truncated normal form is

$$
\mathcal{H}^{3}\left(\mathbf{I}, \phi_{1}\right)=\omega_{1} I_{1}-\frac{1}{2} \omega_{3} I_{2}+\omega_{3} I_{3}+\gamma_{1} I_{2} I_{3}^{1 / 2} \cos \phi_{1},
$$

with $\phi_{1}=2 \theta_{2}+\theta_{3}, \gamma_{1}$ given in Table 8 of Appendix 8 . This normal form is well defined on the resonance curve in II, except on the point obtained as the intersection between the resonance curves related to the vectors $\mathbf{k}^{2}, \mathbf{k}^{6}, \mathbf{k}^{11}$ and $\mathbf{k}^{14}$. It is a point in the parametric plane $(C, A)$ that corresponds to the $3: 1: 2$ resonance. For this case a specific normal 
form should be calculated and will be analysed below when considering $\mathbf{k}^{11}$.

When $\operatorname{dim} S=1$, i.e. in the absence of other resonances, we obtain

$$
S=\left\{\left(0,2 I_{3}, I_{3}\right) \mid I_{3} \geq 0\right\} .
$$

In this situation we take $\mathbf{I}$ in $S \backslash\{\mathbf{0}\}$, arriving at

$$
\mathcal{H}^{3}\left(\mathbf{I}, \phi_{1}\right)=2 \gamma_{1} I_{3}^{3 / 2} \cos \phi_{1}
$$

As $\gamma_{1}$ is not null on the resonance curve $2 \omega_{2}-\omega_{3}=0$, see also Fig. 6 , then $\mathcal{H}^{3}$ has a simple zero as a function of $\phi_{1}$ and therefore, applying Theorem 6 , the equilibrium $P$ is unstable.

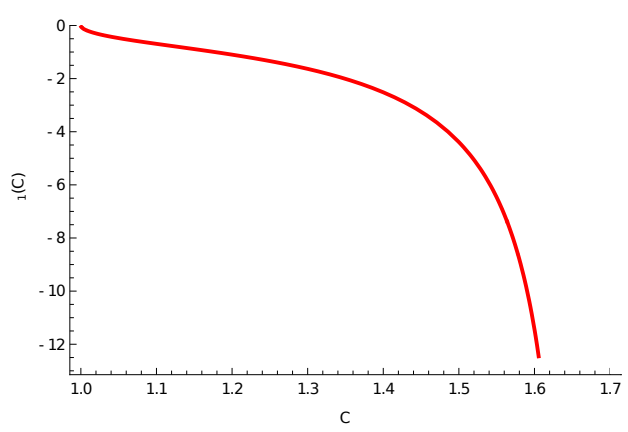

Fig. 6 Graph of $\gamma_{1} \equiv \gamma_{1}(C)$ on the resonance curve $\mathbf{k}^{2}: 2 \omega_{2}-\omega_{3}=0$.

When $\operatorname{dim} S=2$, i.e. when there is an additional resonance, one gets

$$
S=\left\{\left(I_{1}, 2\left(\bar{\omega}_{1} I_{1}+I_{3}\right), I_{3}\right) \mid I_{1}, I_{3} \geq 0\right\} .
$$

In particular, this corresponds to these points: (i) the one obtained from the intersection of the resonance lines related to $\mathbf{k}^{2}$ and $\mathbf{k}^{16}$, thus $H_{2}$ is in 5:1:2 resonance; (ii) the intersection point of the lines related to $\mathbf{k}^{2}$ with $\mathbf{k}^{21}$, and then $H_{2}$ is in $2: 1: 2$ resonance.

Now taking $\mathbf{I}$ in $S \backslash\{\mathbf{0}\}$ we obtain

$$
\mathcal{H}^{3}\left(\mathbf{I}, \phi_{1}\right)=2 \gamma_{1}\left(\bar{\omega}_{1} I_{1}+I_{3}\right) I_{3}^{1 / 2} \cos \phi_{1} .
$$

Applying Theorem 7 the point $P$ is unstable in both situations.

- For $\mathbf{k}^{12}$ the truncated normal form reads as

$$
\begin{aligned}
\mathcal{H}^{4}\left(\mathbf{I}, \phi_{1}\right)= & \omega_{1} I_{1}-\omega_{3} I_{2}+\omega_{3} I_{3}+c_{200} I_{1}^{2}+c_{110} I_{1} I_{2}+c_{101} I_{1} I_{3} \\
& +c_{020} I_{2}^{2}+c_{011} I_{2} I_{3}+c_{002} I_{3}^{2}+\gamma_{3} I_{2} I_{3} \cos \phi_{1},
\end{aligned}
$$

where $\phi_{1}=2 \theta_{2}+2 \theta_{3}$ and $\gamma_{3}, c_{j k l}$ are the coefficients given in Tables 12 and 13 in Appendix 8. The normal form is valid excepting on the two 
points of the parametric plane $(C, A)$ determined as the intersections between the resonance curve associated to $\mathbf{k}^{12}$ with other two resonance curves. For these points specific normal forms should be computed, see details in Fig. 3.

When $\operatorname{dim} S=1$, i.e. in the absence of other resonances, we arrive at

$$
S=\left\{\left(0, I_{3}, I_{3}\right) \mid I_{3} \geq 0\right\} .
$$

Taking $\mathbf{I}$ in $S \backslash\{\mathbf{0}\}$ we get

$$
\begin{aligned}
\mathcal{H}^{4}\left(\mathbf{I}, \phi_{1}\right) & =I_{3}^{2}\left(c_{020}+c_{011}+c_{002}+\gamma_{3} \cos \phi_{1}\right) \\
& \equiv I_{3}^{2}\left(A_{12}+B_{12} \cos \phi_{1}\right) .
\end{aligned}
$$

The coefficient of $I_{3}^{2}$ has a simple zero as a function of $\phi_{1}$ when $\left|A_{12}\right|<$ $\left|B_{12}\right|$ on the resonance curve, which corresponds to $1<C \lesssim 1.16741543$, see the red curve in Fig. 7 (b). Therefore, with the application of Theorem 6 , these values correspond to the point $P$ being unstable. Hamiltonian $\mathcal{H}^{4}\left(\mathbf{I}, \phi_{1}\right) \neq 0$ on the resonance curve for $\mathbf{I} \in S \backslash\{\mathbf{0}\}$ and all $\phi_{1}$ when $1.16741543 \lesssim C \lesssim 1.26357367$. Thence, by the application of Theorem 4 the equilibrium $P$ is Lie stable in these situations, see the blue curve in Fig. 7 (b). When $C \approx 1.16741543$ then $A_{12}+B_{12}=0$ and a higher-order normal form should be calculated.

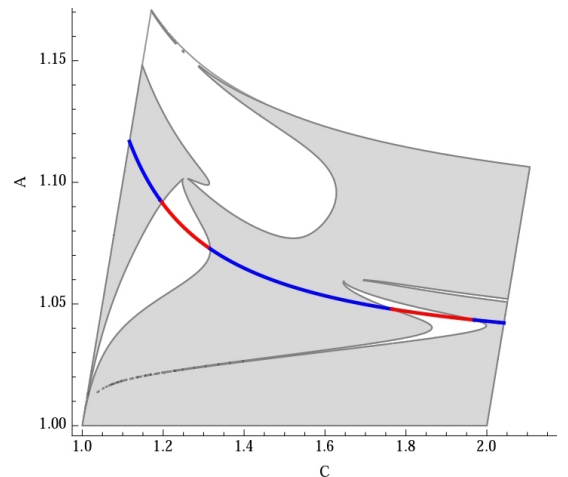

(a) $\mathbf{k}^{11}, A_{11}=c_{200}+3 c_{110}+9 c_{020}, B_{11}=$ $3 \sqrt{3} \gamma_{2}$

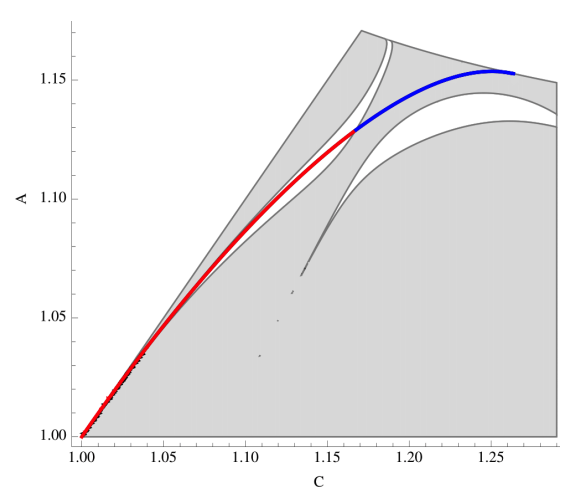

(b) $\mathbf{k}^{12}, A_{12}=c_{020}+c_{011}+c_{002}, B_{12}=\gamma_{3}$

Fig. 7 Curves of instability and Lie stability of the equilibrium point $P$ on the resonance curves defined by $\mathbf{k}^{i} \cdot \omega=0$, with $i=11,12$ inside region II. We consider $A_{i}=A_{i}(C, A), B_{i}=$ $B_{i}(C, A)$, which minimises the computational cost in the calculations. The "islands" in white represent the regions where $\left|A_{i}(C, A)\right|<\left|B_{i}(C, A)\right|$ and the zones in light grey account for $\left|A_{i}(C, A)\right|>\left|B_{i}(C, A)\right|$. Then, we intersect these regions with the resonance curve to obtain the stability and instability intervals. Note that the instability curves (in red) appear as intersections between the white regions and the resonance curves, whereas the Lie stability curves (in blue) are intersections between the light grey regions and the resonance curves.

When $\operatorname{dim} S=2$, there is an additional resonance and we have obtained

$$
S=\left\{\left(I_{1}, \bar{\omega}_{1} I_{1}+I_{3}, I_{3}\right) \mid I_{1}, I_{3} \geq 0\right\} .
$$


In particular this is the intersection of the lines related to $\mathbf{k}^{12}$ with either $\mathbf{k}^{11}$ and $\mathbf{k}^{16}$, that will be handled when we consider $\mathbf{k}^{11}$; and with $\mathbf{k}^{6}$, which corresponds to the $2: 1: 1$ resonance and that requires a specific normal form. In the latter situation the truncation of this normal form evaluated in $S \backslash\{\mathbf{0}\}$ reads as

$$
\mathcal{H}^{3}\left(\mathbf{I}, \phi_{1}\right) \approx 1.10453482 I_{1}^{1 / 2}\left(2 I_{1}+I_{3}\right)^{1 / 2} I_{3}^{1 / 2} \cos \phi_{1},
$$

where $\phi_{1}=\theta_{1}+\theta_{2}-\theta_{3}$. After applying Theorem 6 it is concluded that the point $P$ is unstable.

2. Resonances defined through the following vectors: $\mathbf{k}^{4}=(1,-1,-1)$, so $\omega_{1}+\omega_{2}=\omega_{3}$ and $\bar{\omega}_{1}+\bar{\omega}_{2}=1 ; \mathbf{k}^{6}=(1,1,-1)$, so $\omega_{1}=\omega_{2}+\omega_{3}$ and $\bar{\omega}_{1}-\bar{\omega}_{2}=1 ; \mathbf{k}^{14}=(1,-1,-2)$, so $\omega_{1}+\omega_{2}=2 \omega_{3}$ and $\bar{\omega}_{1}+\bar{\omega}_{2}=2$; and $\mathbf{k}^{16}=(1,1,-2)$, so $\omega_{1}=\omega_{2}+2 \omega_{3}$ and $\bar{\omega}_{1}-\bar{\omega}_{2}=2$.

These resonances belong either to case $\left(a_{1}\right)$ or to case $\left(b_{2}\right)$ with $\mathbf{k} \notin K$. Therefore $\operatorname{dim} S=2$ or $S=\{\mathbf{0}\}$, respectively.

When $S=\{\mathbf{0}\}$, i.e. in the absence of resonances, Lie stability holds from $H_{2}$ by applying Theorem 4 . However, when $\operatorname{dim} S=2$ there is an extra resonance. For example this occurs in the intersection of lines associated to the different $\mathbf{k}^{i}$ :

- $\mathbf{k}^{4}$ and $\mathbf{k}^{11}$, which corresponds to the $3: 1: 4$ resonance and that will be studied when we tackle the case $\mathbf{k}^{11}$.

- $\mathbf{k}^{6}$ with $\mathbf{k}^{12}$, that is the 2:1:1 resonance, and that was analysed when we handled $\mathbf{k}^{12}$. It yields instability.

- $\mathbf{k}^{6}$ with $\mathbf{k}^{2}, \mathbf{k}^{11}$ and $\mathbf{k}^{14}$, that corresponds to the 3:1:2 resonance and that will be analysed when we consider $\mathbf{k}^{11}$.

- $\mathbf{k}^{16}$ with $\mathbf{k}^{2}$, that corresponds to the 5:1:2 resonance and that was analysed when we studied the resonance vector $\mathbf{k}^{2}$; it leads to instability.

- $\mathbf{k}^{16}$ with $\mathbf{k}^{11}$ and $\mathbf{k}^{12}$, that corresponds to the $3: 1: 1$ resonance and that will be seen when we handle $\mathbf{k}^{11}$.

When $\operatorname{dim} S=2$ we compute the normal form, evaluate it at $\mathbf{I} \in S \backslash\{\mathbf{0}\}$ and obtain the following results:

- For vectors $\mathbf{k}^{4}$ and $\mathbf{k}^{6}$, the normal form at order one can be written as

$$
\mathcal{H}^{3}\left(\mathbf{I}, \phi_{1}\right)=f\left(\omega_{1}, \omega_{2}\right) I_{1}^{1 / 2} I_{2}^{1 / 2} I_{3}^{1 / 2} \cos \phi_{1},
$$

where $f\left(\omega_{1}, \omega_{2}\right)$ is well defined in region II for $\mathbf{k}^{4}$ and is well defined for $\mathbf{k}^{6}$ excepting the 3:1:2 resonance, that requires a specific normal form that will be computed when analysing $\mathbf{k}^{11}$. The angle $\phi_{1}$ is either $\theta_{1}-\theta_{2}-\theta_{3}$ for $\mathbf{k}^{4}$ and $\theta_{1}+\theta_{2}-\theta_{3}$ for $\mathbf{k}^{6}$.

In the truncated normal form we set $I_{2}=\left(\bar{\omega}_{1} I_{1}+I_{3}\right) / \bar{\omega}_{2}$ and evaluate the resulting Hamiltonian in $S \backslash\{\mathbf{0}\}$, checking that it changes sign, hence Lie stability cannot be concluded from Theorem 4. Moreover, it is not possible to apply Theorem 7 because the components of $\mathbf{k}^{i}$ $(i=4,6)$ change sign. 
- For vectors $\mathbf{k}^{14}$ and $\mathbf{k}^{16}$, the normal form at order two becomes

$$
\mathcal{H}^{4}\left(\mathbf{I}, \phi_{1}\right)=f_{1} I_{1}^{2}+f_{2} I_{1} I_{3}+f_{3} I_{3}^{2}+f_{4} I_{1}^{1 / 2} I_{2}^{1 / 2} I_{3} \cos \phi_{1},
$$

where the functions $f_{i} \equiv f_{i}\left(\omega_{1}, \omega_{2}\right)$ are well defined in II for $\mathbf{k}^{14}$ and $\mathbf{k}^{16}$ excepting the 3:1:2 resonance (for $\mathbf{k}^{14}$ ) and the 3:1:1 and 5:1:2 resonances (for $\mathbf{k}^{16}$ ). These resonances require specific normal forms. The angle $\phi_{1}$ is $\theta_{1}-\theta_{2}-2 \theta_{3}$ for $\mathbf{k}^{14}$ and $\theta_{1}+\theta_{2}-2 \theta_{3}$ for $\mathbf{k}^{16}$.

In $\mathcal{H}^{4}$ we replace $I_{2}=\left(\bar{\omega}_{1} I_{1}+I_{3}\right) / \bar{\omega}_{2}$, concluding that in contrast with the former case, i.e. the resonance vectors $\mathbf{k}^{4}$ and $\mathbf{k}^{6}$, there are resonances for which the point $P$ is Lie stable and others for which $P$ is unstable.

3. Resonances defined through $\mathbf{k}^{7}=(2,0,-1)$, i.e. $2 \omega_{1}=\omega_{3}$ and $2 \bar{\omega}_{1}=1$; and $\mathbf{k}^{21}=(2,0,-2)$, i.e. $\omega_{1}=\omega_{3}$ and $\bar{\omega}_{1}=1$.

These resonances belong either to case $\left(a_{1}\right)$ or to case $\left(a_{2}\right)$. Therefore, $\operatorname{dim} S=2$ or $S=\{\mathbf{0}\}$, respectively.

When $S=\{\mathbf{0}\}$ Lie stability is concluded from $\mathrm{H}_{2}$ by the application of Theorem 4 , and when $\operatorname{dim} S=2$ there is a double resonance. In this latter case we compute a specific normal form for $\mathbf{k}^{7}$ and another one for $\mathbf{k}^{21}$, evaluate them at $\mathbf{I} \in S \backslash\{\mathbf{0}\}$ and obtain the following:

- For $\mathbf{k}^{7}$ the normal form is

$$
\mathcal{H}^{3}\left(\mathbf{I}, \phi_{1}\right)=f\left(\omega_{1}, \omega_{2}\right) I_{1} I_{3}^{1 / 2} \cos \phi_{1},
$$

where $\phi_{1}=2 \theta_{1}-\theta_{3}, f\left(\omega_{1}, \omega_{2}\right)$ is well defined in region II.

- For $\mathbf{k}^{21}$ we end up with

$$
\mathcal{H}^{4}\left(\mathbf{I}, \phi_{1}\right)=f\left(\omega_{1}, \omega_{2}\right) I_{1} I_{3} \cos \phi_{1},
$$

where $\phi_{1}=2 \theta_{1}-2 \theta_{3}, f\left(\omega_{1}, \omega_{2}\right)$ is well defined in region II excepting the 2:1:2 resonance. This is precisely the intersection point of the resonance lines related to $\mathbf{k}^{21}$ and $\mathbf{k}^{2}$, see Fig. 3. At this point a specific normal form should be computed and, as we have seen in the analysis of $\mathbf{k}^{2}$, it leads to instability.

In conclusion, when $\operatorname{dim} S=2$ instability or Lie stability cannot be established for resonances related to $\mathbf{k}^{7}$ and $\mathbf{k}^{21}$, as the truncated normal form evaluated in $S \backslash\{\mathbf{0}\}$ changes sign, thus Theorem 4 does not apply.

4. Resonance defined through $\mathbf{k}^{11}=(1,3,0)$, so $\omega_{1}=3 \omega_{2}$ and $\bar{\omega}_{1}=3 \bar{\omega}_{2}$.

In this case we are either in $\left(a_{1}\right)$ or in $\left(b_{2}\right)$ with $\mathbf{k} \in K$, thence $\operatorname{dim} S=2$ or $\operatorname{dim} S=1$, respectively. The normal form truncated at order 2 is

$$
\begin{aligned}
\mathcal{H}^{4}\left(\mathbf{I}, \phi_{1}\right)= & 3 \omega_{2} I_{1}-\omega_{2} I_{2}+\omega_{3} I_{3}+c_{200} I_{1}^{2}+c_{110} I_{1} I_{2}+c_{101} I_{1} I_{3} \\
& +c_{020} I_{2}^{2}+c_{011} I_{2} I_{3}+c_{002} I_{3}^{2}+\gamma_{2} I_{1}^{1 / 2} I_{2}^{3 / 2} \cos \phi_{1},
\end{aligned}
$$


where $\phi_{1}=\theta_{1}+3 \theta_{2}$ and $\gamma_{2}, c_{j k l}$ are given in Tables 9, 10 and 11 in Appendix 8. This normal form is well defined excepting at four values, which correspond to the four intersection points of the curve related to the vector $\mathbf{k}^{11}$ with the other resonance curves, see Fig. 3. A specific normal form should be computed at these points.

- When $\operatorname{dim} S=1$, i.e. in the absence of more resonances, one gets

$$
S=\left\{\left(I_{1}, 3 I_{1}, 0\right) \mid I_{1} \geq 0\right\} .
$$

Evaluating $\mathcal{H}^{4}$ in $S \backslash\{\mathbf{0}\}$ we end up with

$$
\begin{aligned}
\mathcal{H}^{4}\left(\mathbf{I}, \phi_{1}\right) & =I_{1}^{2}\left(c_{200}+3 c_{110}+9 c_{020}+3 \sqrt{3} \gamma_{2} \cos \phi_{1}\right) \\
& \equiv I_{1}^{2}\left(A_{11}+B_{11} \cos \phi_{1}\right) .
\end{aligned}
$$

If the coefficient of $I_{1}^{2}$ has a simple zero as a function of $\phi_{1}$ then, applying Theorem 6 , the equilibrium $P$ is unstable. This occurs when $\left|A_{11}\right|<$ $\left|B_{11}\right|$ on the resonance curve, which corresponds to $1.19633123 \lesssim C \lesssim$ 1.31669778 and $1.76531759 \lesssim C \lesssim 1.96884828$, see the red curves in Fig. 7 (a). Hamiltonian $\mathcal{H}^{4}\left(\mathbf{I}, \phi_{1}\right) \neq 0$ on the resonance curve for $\mathbf{I} \in$ $S \backslash\{\mathbf{0}\}$ and all $\phi_{1}$ when $1.11679395 \lesssim C \lesssim 1.19633123,1.31669778 \lesssim$ $C \lesssim 1.76531759$ and $1.96884828 \lesssim C \lesssim 2.04222818$. In this situation, by the application of Theorem 4 the equilibrium $P$ is Lie stable, see the blue curves in Fig. 7 (a). When $C \approx 1.31669778,1.76531759,1.96884828$ a higher order analysis is required because $\left|A_{11}\right|+\left|B_{11}\right|=0$. The value $C \approx 1.19633123$ corresponds to the intersection point of the resonance lines associated to $\mathbf{k}^{2}, \mathbf{k}^{6}, \mathbf{k}^{11}$ and $\mathbf{k}^{14}$, i.e. the $3: 1: 2$ resonance, that will be considered below.

- When $\operatorname{dim} S=2$, as it corresponds to a case of double resonance, we arrive at the following possibilities:

- The intersection of the resonance lines associated to $\mathbf{k}^{11}$ with $\mathbf{k}^{12}$ and $\mathbf{k}^{16}$, which corresponds to the 3:1:1 resonance. The truncated normal form is

$$
\begin{aligned}
\mathcal{H}^{4}\left(\mathbf{I}, \phi_{1}\right)= & 3 \omega_{2} I_{1}-\omega_{2} I_{2}+\omega_{2} I_{3}+c_{200} I_{1}^{2}+c_{110} I_{1} I_{2}+c_{101} I_{1} I_{3} \\
& +c_{020} I_{2}^{2}+c_{011} I_{2} I_{3}+c_{002} I_{3}^{2}+\gamma_{1} I_{1}^{1 / 2} I_{2}^{3 / 2} \cos \phi_{1} \\
& +\gamma_{2} I_{1}^{1 / 2} I_{2}^{1 / 2} I_{3} \cos \phi_{2}+\gamma_{3} I_{2} I_{3} \cos \left(\phi_{1}-\phi_{2}\right),
\end{aligned}
$$

where $\phi_{1}=\theta_{1}+3 \theta_{2}, \phi_{2}=\theta_{1}+\theta_{2}-2 \theta_{3}$ and the coefficients $\omega_{i}, \gamma_{j}$ and $c_{i j k}$ are real numbers. The Hamiltonian evaluated in $S \backslash\{\mathbf{0}\}$ changes sign and then Lie stability cannot be deduced from Theorem 4.

- The intersection of the lines related to $\mathbf{k}^{11}$ with $\mathbf{k}^{2}, \mathbf{k}^{6}$ and $\mathbf{k}^{14}$, that corresponds to the $3: 1: 2$ resonance. The truncated normal form evaluated in $S \backslash\{\mathbf{0}\}$ with floating-point approximation reads as

$$
\begin{aligned}
\mathcal{H}^{3}(\mathbf{I}, \phi) \approx & 0.80806060 I_{1}^{1 / 2} I_{2}^{1 / 2} I_{3}^{1 / 2} \cos \phi_{1} \\
& +0.54123511 I_{2} I_{3}^{1 / 2} \cos \phi_{2}
\end{aligned}
$$


with $\phi=\left(\phi_{1}, \phi_{2}\right)=\left(\theta_{1}+\theta_{2}-\theta_{3}, 2 \theta_{2}-\theta_{3}\right)$. Applying Theorem 2.1 in [31] the equilibrium is unstable, see also [32], [6].

- The intersection of the lines obtained from $\mathbf{k}^{11}$ with $\mathbf{k}^{21}$, that is the $3: 1: 3$ resonance. The specific truncated normal form is

$$
\begin{aligned}
\mathcal{H}^{4}(\mathbf{I}, \phi)= & 3 \omega_{2} I_{1}-\omega_{2} I_{2}+\omega_{2} I_{3}+c_{200} I_{1}^{2}+c_{110} I_{1} I_{2}+c_{101} I_{1} I_{3} \\
& +c_{020} I_{2}^{2}+c_{011} I_{2} I_{3}+c_{002} I_{3}^{2}+\gamma_{1} I_{1}^{1 / 2} I_{2}^{3 / 2} \cos \phi_{1} \\
& +\gamma_{2} I_{1} I_{3} \cos \phi_{2},
\end{aligned}
$$

where $\phi=\left(\phi_{1}, \phi_{2}\right)$ with $\phi_{1}=\theta_{1}+3 \theta_{2}, \phi_{2}=2 \theta_{1}-2 \theta_{3}$ and the coefficients are numbers. The Hamiltonian evaluated in $S \backslash\{\mathbf{0}\}$ changes sign, thence Lie stability cannot be obtained from Theorem 4 .

- The intersection of the lines associated to $\mathbf{k}^{11}$ with $\mathbf{k}^{4}$, that is the 3:1:4 resonance. The truncated normal form evaluated in $S \backslash\{\mathbf{0}\}$ with floating-point approximation is

$$
\mathcal{H}^{3}\left(\mathbf{I}, \phi_{1}\right) \approx 1.20430590 I_{1}^{1 / 2} I_{2}^{1 / 2} I_{3}^{1 / 2} \cos \phi_{1},
$$

where $\phi_{1}=\theta_{1}-\theta_{2}-\theta_{3}$. The Hamiltonian evaluated in $S \backslash\{\mathbf{0}\}$ changes sign and then Theorem 4 cannot be used to get Lie stability.

- In the remaining cases with $\operatorname{dim} S=2$ there are resonances associated to Lie stable cases, such as the 15:5:4, whereas others are related to unstable ones, for instance the 12:4:9, 9:3:10, 60:20:97, 30:10:51 and 15:5:27 resonances.

Remark 3 The expressions of $f\left(\omega_{1}, \omega_{2}\right)$ and $f_{i}\left(\omega_{1}, \omega_{2}\right)$ in items 2 and 3 as well as the numerical coefficients of the normal form Hamiltonians $\mathcal{H}^{4}(\mathbf{I}, \phi)$ corresponding to the cases with $\operatorname{dim} S=2$ in item 4 can be provided by the first author.

Remark 4 The computations performed in the previous analysis are done in rational arithmetic, that is, without resorting to floating-point approximations. However since some numerical expressions become huge we have opted by representing them using eight digits.

We summarise the results in the following.

Theorem 1 For $(C, A) \in I I$ the equilibrium $P$ associated to the Hamiltonian system related to (3) is Lie stable in the following cases (see details in Fig. 4):

- In the dark-blue region.

- In the light-blue region when

$\left(a_{2}\right) \bar{\omega}_{1} \in \mathbb{Q}, \bar{\omega}_{2} \in \mathbb{R} \backslash \mathbb{Q}$

$\left(b_{1}\right) \bar{\omega}_{1}, \bar{\omega}_{2} \in \mathbb{R} \backslash \mathbb{Q}, \nexists \mathbf{k} ;$ 
$\left(b_{2}\right) \bar{\omega}_{1}, \bar{\omega}_{2} \in \mathbb{R} \backslash \mathbb{Q}, \exists \mathbf{k} \notin K$

$\left(b_{3}\right) \bar{\omega}_{1} \in \mathbb{R} \backslash \mathbb{Q}, \bar{\omega}_{2} \in \mathbb{Q}$.

- On the resonance lines associated to $\mathbf{k}^{4}, \mathbf{k}^{6}, \mathbf{k}^{7}, \mathbf{k}^{14}, \mathbf{k}^{16}$ and $\mathbf{k}^{21}$ in the absence of other resonances.

- On the resonance lines associated to $\mathbf{k}^{11}$ and $\mathbf{k}^{12}$ : for certain double resonances and in the blue parts of lines in Fig. 7 in the absence of further resonances.

- On the resonance lines associated to $\mathbf{k}^{14}$ and $\mathbf{k}^{16}$ for certain double resonances.

The equilibrium $P$ is unstable in the following cases:

- On the resonance curve related to $\mathbf{k}^{2}$.

- On the resonance curves related to $\mathbf{k}^{11}$ and $\mathbf{k}^{12}$ : for certain double resonances and in the red parts of lines in Fig. 7 in the absence of further resonances.

- On the intersection of the resonance lines related to $\mathbf{k}^{6}$ and $\mathbf{k}^{12}$, i.e. the 2:1:1 resonance.

- On the resonance curves related to $\mathbf{k}^{14}$ and $\mathbf{k}^{16}$ for certain double resonances.

Remark 5 As we have pointed out when performing the analysis to accomplish Theorem 1, there are some cases that are more likely to be unstable. The reason is that Hamiltonian function changes sign, thus Lie stability cannot be accomplished from part (A) of Theorem 4, but part (B) of the same theorem suggests that we could achieve instability for the equilibrium point. However we would need to add extra hypotheses to the higher-order terms in normal form in order to build a suitable Chetaev function needed to get instability. These situations are:

- The intersection of the resonance curves associated to $\mathbf{k}^{4}$ and $\mathbf{k}^{11}$, i.e. the $3: 1: 4$ resonance.

- The intersection of the resonance curves associated to $\mathbf{k}^{11}, \mathbf{k}^{12}$ and $\mathbf{k}^{16}$, i.e. the $3: 1: 1$ resonance.

- The intersection of the resonance curves associated to $\mathbf{k}^{11}$ and $\mathbf{k}^{21}$, i.e. the $3: 1: 3$ resonance.

- Double resonances associated to the resonance lines related to the vectors $\mathbf{k}^{4}, \mathbf{k}^{6}, \mathbf{k}^{7}$ and $\mathbf{k}^{21}$.

There are cases that require the computation of a higher-order normal form Hamiltonian, namely,

- The case $\beta_{3}=0$ and $\bar{\omega}_{1} \in \mathbb{R} \backslash \mathbb{Q}, \bar{\omega}_{2} \in \mathbb{Q}$ (see Fig. 5).

- On the resonance line related to the vector $\mathbf{k}^{11}$, the values that correspond to $\left|A_{11}\right|+\left|B_{11}\right|=0$, i.e. $C \approx 1.31669778,1.76531759,1.96884828$.

- On the resonance line related to the vector $\mathbf{k}^{12}$ the value $C \approx 1.16141543$, which corresponds to the identity $A_{12}+B_{12}=0$. 
Remark 6 All cases of Theorem 1 related to double resonances form infinite although countable (i.e. discrete) sets, and in the parametric plane $(C, A)$ they always appear as isolated points.

In Tables 14 and 15 the resonant cases of orders 3 and 4 corresponding to $\operatorname{dim} S=0$ and $\operatorname{dim} S=1$ are listed, respectively, in the absence of more resonances. Both tables appear in Appendix 8. There, together with the resonance vectors, we find the first integrals $F_{i}$ with the corresponding coefficients $\sigma$ that are used to express $H_{2}$ as a linear combination of $F_{i}$ as written in formula (23). Note that $\sigma$ is always a Diophantine vector.

Remark 7 Sufficiently close to the resonance lines of Fig. 3, the normal form Hamiltonian (10) cannot be used when the vector $\sigma$ is not Diophantine, as in the process of the transformation to normal form small denominators arise in some terms of the generating functions and in $\mathcal{H}_{4}$, deteriorating the calculations. Then, we can apply a detuning argument and vary the parameters $\omega_{i}$ adequately. We notice that it is enough to introduce two small parameters, say $\delta_{1}, \delta_{2}$, in such a way that for the resulting quadratic term $H_{2}$ one always has $\operatorname{dim} S=0$ or 1 , due to the density character of these cases in the parametric plane $(C, A)$. When $\operatorname{dim} S=0$ one readily obtains Lie stability. When $\operatorname{dim} S=1$, because of the detuning process, considering $I \in S \backslash\{\mathbf{0}\}$, the leading term of the perturbation is of the form $\bar{h}\left(\delta_{1}, \delta_{2}\right) I_{j}$ with $j$ either 1,2 or 3 (as terms of orders three and higher are always of smaller size). Without exception we can take $\bar{h} \neq 0$, yielding Lie stability in all cases.

\section{Asymptotic estimates}

To determine bounds on the solution and time for the Lie stable equilibria we apply Corollary 1, given in Appendix 7, to bound over exponentially long times the solutions corresponding to the equilibria when they are Lie stable.

In our study the solution is expressed as $\mathbf{I}(t)$, that is a function of the order of $|\mathbf{x}|^{2}$. So, $|\mathbf{x}(0)| \sim \varepsilon$ implies $|\mathbf{I}(0)| \sim \varepsilon^{2}$. Introducing $\epsilon=\varepsilon^{2}$ and setting $\mathbf{I}_{0}=\mathbf{I}(0)$, then the thesis of Corollary 1 reads as

$$
|\mathbf{I}(t)|<\alpha \epsilon^{j^{\prime} / j} \quad \text { for all } t \text { with } \quad 0 \leq t \leq T=\mathcal{C} \exp \left(\frac{\mathcal{E}}{\epsilon^{1 /(2(\nu+1))}}\right),
$$

where $\alpha, \mathcal{C}, \mathcal{E}$ are obtained respectively from $a, C$ and $E$ appearing in Theorem 5 or Corollary 1.

On the one hand, the solution's bound depends on the order $j$ in the normal form (7) that determines the Lie stability. The lower the order, the better, as the solution is closer to the initial point. When $S=\{\mathbf{0}\}$, then $j^{\prime}=j=2$ and in the rest of situations addressed in this paper, $j^{\prime}=j=4$ as Lie stability cannot be reached with $j=3$. For the degenerate cases where $\mathcal{H}^{4}$ is not enough to conclude Lie stability one should reach at least order 6 in order to obtain more Lie stable cases, thus $j=6$. (Note that we have not tackled these degeneracies in our analysis.) 
On the other hand, the time validity depends on the number of independent first integrals, that is $d$, because vector $\sigma=\left(\sigma_{1}, \ldots, \sigma_{d}\right)$ satisfies the Diophantine condition with $\nu \geq d-1$ (see Definition 3 in Appendix 7). The number of independent first integrals ranges from 1 to 3 in the problem at hand. The lower the number of integrals, the better the estimate, because the time validity is longer. If $d=1$ then no Diophantine assumption is required. In Table 2 we give $j^{\prime}, j$ and $d$ for each Lie stable case in the absence of resonances of orders 3 and 4 . Table 3 accounts for the same data on the resonance curves appearing in Fig. 3, excluding the intersection points.

\begin{tabular}{|l|c|c|}
\hline Case & $j^{\prime} / j$ & $d$ \\
\hline$\left(a_{1}\right) \bar{\omega}_{1}, \bar{\omega}_{2} \in \mathbb{Q}$ & 4 & 1 \\
\hline$\left(a_{2}\right) \bar{\omega}_{1} \in \mathbb{Q}, \bar{\omega}_{2} \in \mathbb{R} \backslash \mathbb{Q}$ & 2 & 2 \\
\hline$\left(b_{1}\right) \bar{\omega}_{1}, \bar{\omega}_{2} \in \mathbb{R} \backslash \mathbb{Q} \& \nexists \mathbf{k}$ & 2 & 3 \\
\hline$\left(b_{2}\right) \bar{\omega}_{1}, \bar{\omega}_{2} \in \mathbb{R} \backslash \mathbb{Q} \& \exists \mathbf{k} \in K$ & 4 & 2 \\
\hline$\left(b_{2}\right) \bar{\omega}_{1}, \bar{\omega}_{2} \in \mathbb{R} \backslash \mathbb{Q} \& \exists \mathbf{k} \notin K$ & 2 & 2 \\
\hline$\left(b_{3}\right) \bar{\omega}_{1} \in \mathbb{R} \backslash \mathbb{Q}, \bar{\omega}_{2} \in \mathbb{Q}$ & 4 & 2 \\
\hline
\end{tabular}

Table 2 Values of the parameters involved in the estimates: $j^{\prime}$ (lowest degree of the perturbating Hamiltonian), $j$ (degree that determines Lie stability) and $d$ (number of linearly independent integrals) in each case in the absence of resonances of orders 3 and 4 . Vector $\mathbf{k}$ is a resonance vector.

\begin{tabular}{|c|c|c|}
\hline Resonance & $j^{\prime} / j$ & $d$ \\
\hline $\mathbf{k}^{12}$ & 4 & $\begin{array}{l}\left(a_{1}\right): 1 \\
\left(b_{3}\right): 2 \\
\end{array}$ \\
\hline $\left.\begin{array}{l}\mathbf{k}^{4} \\
\mathbf{k}^{6}\end{array}\right\}$ & $\left(b_{2}\right) \exists \mathbf{k} \notin K: 2$ & $\left(b_{2}\right) \exists \mathbf{k} \notin K: 2$ \\
\hline $\left.\begin{array}{l}\mathbf{k}^{14} \\
\mathbf{k}^{16}\end{array}\right\}$ & $\begin{array}{r}\left(a_{1}\right): 4 \\
\left(b_{2}\right) \exists \mathbf{k} \notin K: 2\end{array}$ & $\begin{array}{r}\left(a_{1}\right): 1 \\
\left(b_{2}\right) \exists \mathbf{k} \notin K: 2\end{array}$ \\
\hline $\left.\begin{array}{l}\mathbf{k}^{7} \\
\mathbf{k}^{21}\end{array}\right\}$ & $\left(a_{2}\right): 2$ & $\left(a_{2}\right): 2$ \\
\hline $\mathbf{k}^{11}$ & 4 & $\begin{array}{r}\left(a_{1}\right): 1 \\
\left(b_{2}\right) \exists \mathbf{k} \in K: 2 \\
\end{array}$ \\
\hline
\end{tabular}

Table 3 Values of the parameters involved in the estimates: $j^{\prime}$ (lowest degree of the perturbing Hamiltonian), $j$ (degree that determines Lie stability) and $d$ (number of linearly independent integrals) in the Lie stable cases corresponding to the resonances of orders 3 and 4 , excluding the intersection points.

The main result in this section is the following. 
Theorem 2 For the Hamiltonian system associated with (3), in the cases where the equilibrium $P$ is Lie stable and $(C, A) \in I I$ (excepting the resonance curves):

1. When $\bar{\omega}_{1}, \bar{\omega}_{2} \in \mathbb{Q}$, then there exist $\alpha>1, \mathcal{C}>0, \mathcal{E}>0$ and $\epsilon_{0}>0$ such that for all $\epsilon \in\left(0, \epsilon_{0}\right)$ with $\left|\mathbf{I}_{0}\right|<\epsilon$ :

$$
|\mathbf{I}(t)|<\alpha \epsilon \quad \text { for all } t \text { with } \quad 0 \leq t \leq T=\mathcal{C} \exp \left(\frac{\mathcal{E}}{\epsilon^{1 / 2}}\right)
$$

2. When $\sigma$ is Diophantine, then there exist $\alpha>1, \mathcal{C}>0, \mathcal{E}>0$ and $\epsilon_{0}>0$ such that for all $\epsilon \in\left(0, \epsilon_{0}\right)$ with $\left|\mathbf{I}_{0}\right|<\epsilon$ :

(a) If either $\bar{\omega}_{1} \in \mathbb{Q}$ and $\bar{\omega}_{2} \in \mathbb{R} \backslash \mathbb{Q}$ or $\bar{\omega}_{1} \in \mathbb{R} \backslash \mathbb{Q}$ and $\bar{\omega}_{2} \in \mathbb{Q}$ or $\bar{\omega}_{1}, \bar{\omega}_{2} \in \mathbb{R} \backslash \mathbb{Q}$ and there is $\mathbf{k} \in \mathbb{Z}^{3}$ satisfying (13), then

$$
|\mathbf{I}(t)|<\alpha \epsilon \quad \text { for all } t \quad \text { with } \quad 0 \leq t \leq T=\mathcal{C} \exp \left(\frac{\mathcal{E}}{\epsilon^{1 / 4}}\right)
$$

(b) If $\bar{\omega}_{1}, \bar{\omega}_{2} \in \mathbb{R} \backslash \mathbb{Q}$ and there is no $\mathbf{k} \in \mathbb{Z}^{3}$ satisfying (13), then

$$
|\mathbf{I}(t)|<\alpha \epsilon \quad \text { for all } t \text { with } \quad 0 \leq t \leq T=\mathcal{C} \exp \left(\frac{\mathcal{E}}{\epsilon^{1 / 6}}\right)
$$

Remark 8 For the resonance lines represented in Fig. 31 , the estimates in the Lie stable cases are obtained by replacing in (20) the values appearing in Table 3 for each situation, setting $\nu=d-1$ in Definition 3.

Notice that the constants $\alpha, \mathcal{C}$ and $\mathcal{E}$ do not depend on $\epsilon$ and are calculated using bounds on the normal-form terms.

\section{Quasi-periodic solutions and KAM 3-tori}

In the following we carry out the existence of 3-dimensional KAM tori and quasi-periodic motions encasing the equilibrium $P$ of the Hamiltonian system related to (3). For this we start by applying both the classical Kolgomorov theorem and the extension due to Arnold, see the details in Chapter 6 in [1]. We consider the truncated normal form $\mathcal{H}^{4}$ determined by Hamiltonian (11) 
and compute the determinants

$$
\begin{aligned}
D_{3}= & \left|\frac{\partial^{2} \mathcal{H}^{4}}{\partial \mathbf{I}^{2}}\right|_{\mathbf{I}=\mathbf{0}} \\
= & -2\left(c_{200}\left(c_{011}^{2}-4 c_{020} c_{002}\right)+c_{110}^{2} c_{002}-c_{110} c_{101} c_{011}+c_{101}^{2} c_{020}\right), \\
D_{4}= & \left|\begin{array}{ll}
\frac{\partial^{2} \mathcal{H}^{4}}{\partial \mathbf{I}^{2}} & \frac{\partial \mathcal{H}^{4}}{\partial \mathbf{I}} \\
\frac{\partial \mathcal{H}^{4}}{\partial \mathbf{I}} & 0
\end{array}\right|_{\mathbf{I}=\mathbf{0}} \\
= & \omega_{1}^{2}\left(c_{011}^{2}-4 c_{020} c_{002}\right)+\omega_{2}^{2}\left(c_{101}^{2}-4 c_{200} c_{002}\right)+\omega_{3}^{2}\left(c_{110}^{2}-4 c_{200} c_{020}\right) \\
& +2 \omega_{1} \omega_{2}\left(c_{101} c_{011}-2 c_{110} c_{002}\right)+2 \omega_{1} \omega_{3}\left(2 c_{101} c_{020}-c_{110} c_{011}\right) \\
& +2 \omega_{2} \omega_{3}\left(c_{110} c_{101}-2 c_{200} c_{011}\right) .
\end{aligned}
$$

Note that the function $D_{3} \equiv D_{3}(C, A)$ is null on the white curves and $D_{4} \equiv$ $D_{4}(C, A)$ is null on the black lines in Fig. 8.

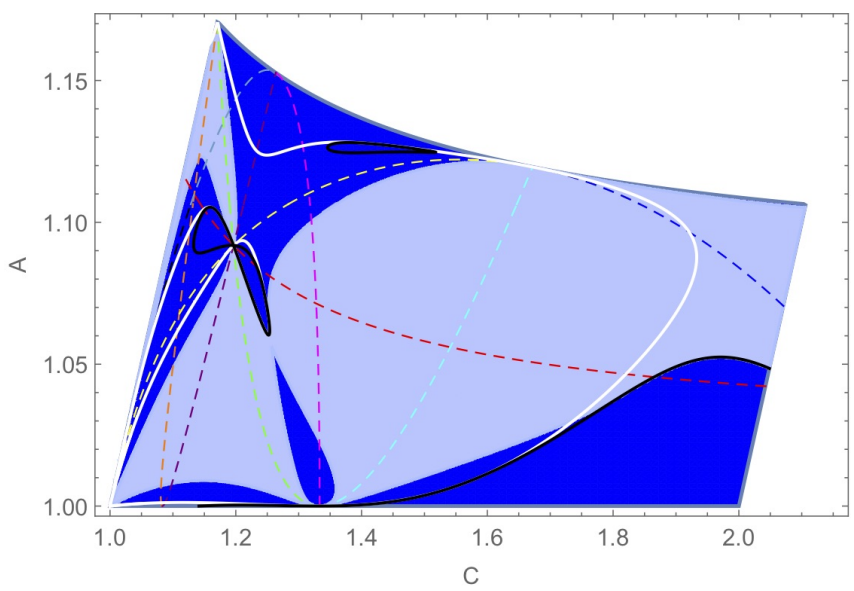

Fig. 8 KAM tori: The white lines correspond to $D_{3}(C, A)=0$ and the black ones account for $D_{4}(C, A)=0$. The dashed colour lines stand for the resonances appearing in Fig. 3.

Therefore, we end up with the following result.

Theorem 3 For $(C, A) \in I I \backslash\left\{\mathbf{k}^{2} \cdot \omega=0, \mathbf{k}^{4} \cdot \omega=0, \mathbf{k}^{6} \cdot \omega=0, \mathbf{k}^{7} \cdot \omega=\right.$ $\left.0, \mathbf{k}^{11} \cdot \omega=0, \mathbf{k}^{12} \cdot \omega=0, \mathbf{k}^{14} \cdot \omega=0, \mathbf{k}^{16} \cdot \omega=0, \mathbf{k}^{21} \cdot \omega=0\right\}$ :

- when $D_{3} \neq 0$, i.e. when Hamiltonian $\mathcal{H}^{4}$ determined by (11) is non-degenerate, or

- when $D_{4} \neq 0$, i.e. when Hamiltonian $\mathcal{H}^{4}$ determined by (11) is isoenergetically non-degenerate, 
then the Hamiltonian (3) is of general elliptic type and under a sufficiently small Hamiltonian perturbation, most of the non-resonant invariant 3-tori $\mathbf{I}=$ const. will, slightly deformed, persist for any sufficiently small perturbation of them, that is, for the full system (3). Moreover, the Lebesgue measure of the complement of the set of tori in the polydisc $|\mathbf{I}|<\varepsilon$ tends to zero when the perturbation is small. More precisely, these invariant tori form a majority in the sense that the measure of the complement of their union is of the order $\mathcal{O}\left(\varepsilon^{1 / 4}\right)$ and can be refined to $\mathcal{O}\left(\varepsilon^{(l-3) / 4}\right)$ when the frequency vector $\omega$ does not satisfy resonance relations of order $l$ with $l>4$. Furthermore, when the frequency vector $\omega$ is Diophantine the estimate becomes exponentially small of order $\mathcal{O}\left(\exp \left(-c^{-1} / \varepsilon^{\alpha}\right)\right)$ for suitable constants $c, \alpha>0$. In the case of isoenergetic non-degeneracy the invariant tori form a majority on each energy-level manifold.

To achieve the above result we have applied Theorem 6.23 together with Remark 6.20 in [1].

Remark 9 When the Hamiltonian system is degenerate and isoenergetically degenerate, the classical KAM theorems do not apply. Nevertheless, under certain conditions we can resort to a theorem by Han, Li and Yi [18] and the previous result can be extended. As an example, consider the following values for the parameters

$$
C \approx 1.56294188, \quad A \approx 1.00803422 .
$$

In this case $D_{3}=0$ and $D_{4}=0$, thus it corresponds to one of the points that belong to the black and yellow curves in Fig. 8. Our plan is to prove the persistence of 3 -tori from $\mathcal{H}^{4}$. The coefficients $c_{j k l}$ for the specific values of $C$ and $A$ given above appear in Table 16 of Appendix 8, they have been obtained from the normal form coefficients calculated in (11). We perform the usual symplectic stretching of coordinates $(\mathbf{I}, \theta) \rightarrow\left(\varepsilon^{2} \mathbf{I}, \theta\right)$ with associated multiplier $\varepsilon^{-2}$ and collect terms in $\varepsilon$ reaching

$$
H(\mathbf{I}, \theta)=\eta_{0}\left(I_{1}, I_{2}, I_{3}\right)+\varepsilon^{2} \eta_{1}\left(I_{1}, I_{2}, I_{3}\right)+\mathcal{O}\left(\varepsilon^{4}\right),
$$

where $\eta_{0}=H_{2}, \eta_{1}=\mathcal{H}_{4}$. Following the notation in [18]: $n=3, a=1, m_{1}=2$, $n_{0}=n_{1}=3, y^{n_{0}}=y^{n_{1}}=\left(I_{1}, I_{2}, I_{3}\right), \widehat{y}^{n_{0}}=\widehat{y}^{n_{1}}=\left(I_{1}, I_{2}, I_{3}\right)$. (Notice that the appearance of $\theta$ in $H$ occurs at order $\mathcal{O}\left(\varepsilon^{4}\right)$.) In this case, the vector of frequencies has dimension 6 and is given by

$$
\Omega=\left(\Omega_{1}, \Omega_{2}, \Omega_{3}, \Omega_{4}, \Omega_{5}, \Omega_{6}\right)=\left(\frac{\partial \eta_{0}}{\partial I_{1}}, \frac{\partial \eta_{0}}{\partial I_{2}}, \frac{\partial \eta_{0}}{\partial I_{3}}, \frac{\partial \eta_{1}}{\partial I_{1}}, \frac{\partial \eta_{1}}{\partial I_{2}}, \frac{\partial \eta_{1}}{\partial I_{3}}\right) .
$$


The corresponding $6 \times 4$-matrix

$$
M_{\Omega}=\left(\begin{array}{cccc}
\Omega_{1} & \frac{\partial \Omega_{1}}{\partial I_{1}} & \frac{\partial \Omega_{1}}{\partial I_{2}} & \frac{\partial \Omega_{1}}{\partial I_{3}} \\
\Omega_{2} & \frac{\partial \Omega_{2}}{\partial I_{1}} & \frac{\partial \Omega_{2}}{\partial I_{2}} & \frac{\partial \Omega_{2}}{\partial I_{3}} \\
\Omega_{3} & \frac{\partial \Omega_{3}}{\partial I_{1}} & \frac{\partial \Omega_{3}}{\partial I_{2}} & \frac{\partial \Omega_{3}}{\partial I_{3}} \\
\Omega_{4} & \frac{\partial \Omega_{4}}{\partial I_{1}} & \frac{\partial \Omega_{4}}{\partial I_{2}} & \frac{\partial \Omega_{4}}{\partial I_{3}} \\
\Omega_{5} & \frac{\partial \Omega_{5}}{\partial I_{1}} & \frac{\partial \Omega_{5}}{\partial I_{2}} & \frac{\partial \Omega_{5}}{\partial I_{3}} \\
\Omega_{6} & \frac{\partial \Omega_{6}}{\partial I_{1}} & \frac{\partial \Omega_{6}}{\partial I_{2}} & \frac{\partial \Omega_{6}}{\partial I_{3}}
\end{array}\right)
$$

evaluated at $\mathbf{I}=\mathbf{0}$ has rank 3 as the maximum minor in absolute value of order 3 is approximately 2.90804425. Therefore, Han, Li and Yi's Theorem guarantees the existence of KAM 3-tori. These invariant tori form a majority in the sense that the measure of the complement of their union is of the order $\mathcal{O}\left(\varepsilon^{\delta}\right)$ with a fixed value of $\delta$ such that $0<\delta<1 / 5$. Pushing the normal form calculation to higher order we would improve this estimate but it has not been done in this paper.

Remark 10 There are invariant tori associated to some unstable elliptic equilibria. For example, consider

$$
C \approx 1.28077074, \quad A \approx 1.04523803
$$

For these values there is a fifth-order resonance relation $4 \omega_{2}=\omega_{3}$. The truncated normal form to order three is given by the Hamiltonian function

$$
\mathcal{H}^{5}\left(\mathbf{I}, \phi_{1}\right)=H_{2}(\mathbf{I})+\mathcal{H}_{4}(\mathbf{I})+\mathcal{H}_{5}\left(\mathbf{I}, \phi_{1}\right)
$$

where

$$
\begin{aligned}
\mathcal{H}_{4}(\mathbf{I}) & =c_{200} I_{1}^{2}+c_{110} I_{1} I_{2}+c_{020} I_{2}^{2}+c_{101} I_{1} I_{3}+c_{011} I_{2} I_{3}+c_{002} I_{3}^{2}, \\
\mathcal{H}_{5}\left(\mathbf{I}, \phi_{1}\right) & =d I_{2}^{2} I_{3}^{1 / 2} \sin \phi_{1},
\end{aligned}
$$

with $\phi_{1}=4 \theta_{2}+\theta_{3}$ and $c_{j k l}, d$ have been written in Table 17 of Appendix 8 . Specfically, the $c_{j k l}$ in the table have been determined by using the coefficients $c_{j k l}$ of (11), replacing $\omega_{i}$ conveniently whereas $d$ is derived from the normal form terms of order three. In this case, the resonance vector is $\mathbf{k}=(0,4,1)$; $F_{1}=-I_{2}+4 I_{3}$ and $F_{2}=I_{1}$ are the corresponding first integrals and, in the absence of other resonances, the set $S$ is given by $\left\{\left(0,4 I_{3}, I_{3}\right) \mid I_{3} \geq 0\right\}$, thus $\operatorname{dim} S=1$. Considering $\mathbf{I} \in S \backslash\{\mathbf{0}\}: \mathcal{H}^{4}(\mathbf{I})=0$ and $\mathcal{H}_{5}\left(\mathbf{I}, \phi_{1}\right)$ has a simple zero as a function of $\phi_{1}$. Then, using Theorem 6 we conclude that the equilibrium is unstable. Nevertheless,

$$
\left|\frac{\partial^{2} \mathcal{H}^{4}(\mathbf{I})}{\partial \mathbf{I}^{2}}\right|_{\mathbf{I}=\mathbf{0}} \approx-0.10557520 .
$$

Therefore, the Hamiltonian is non-degenerate and Theorem 6.23 in [1] leads to the existence of invariant KAM tori and quasi-periodic solutions. 


\section{Concluding remarks}

1. This work extends the results about non-linear stability appearing in the literature. On the one hand, Markeev and Sokolskii [24] prove the formal stability in the hatched region in their Fig. 3 (although there is a misprint and they say instability). Their formal stability region corresponds to our dark blue region in Fig. 4. With respect to the non-hatched region, they explain that the analysis of $\mathcal{H}^{6}$ would be required. With the approach followed in the present paper, we have obtained Lie stability of a dense set of points in our light-blue region with the analysis of $\mathcal{H}^{4}$ as it is stated in Theorem 1.

On the other hand, these authors establish the stability for the majority of initial conditions off the resonance curves where $D_{3}$ or $D_{4}$ are non-zero. In other words, they prove the existence of invariant tori in the region where $D_{3}$ or $D_{4}$ are non-zero. This is our Fig. 8, that is not given in [24].

2. We also generalise the stability results on the resonance curves. Specifically, we conclude Lie stability in the cases where [24] establish that they cannot be unstable. In the resonant cases $\mathbf{k}^{11}$ and $\mathbf{k}^{12}$ we obtain Lie stability for the full Hamiltonian, while Markeev and Sokolskii [24] only achieve stability for the truncated Hamiltonian system up to terms of degree 4 in rectangular coordinates.

3. The present study also deals with double resonances. In fact, case $\left(a_{1}\right)$, i.e. $\bar{\omega}_{1}, \bar{\omega}_{2} \in \mathbb{Q}$, corresponds to double resonances. We achieve Lie stability for all double resonances in the dark blue region and also some double resonances related with the resonance vectors $\mathbf{k}^{11}, \mathbf{k}^{12}, \mathbf{k}^{14}$ and $\mathbf{k}^{16}$.

4. One of the strengths of Theorem 4 is that it allows one to decide on Lie stability through Hamiltonians depending on resonant angles. For instance, this is the case of resonances related to the vectors $\mathbf{k}^{11}$ and $\mathbf{k}^{12}$, whose stability is accomplished through $\mathcal{H}^{4}(\mathbf{I}, \phi)$, see Fig. 7 .

5. In addition to the study of the Lie stability we bound the solutions on exponentially long times, see Theorem 2. This type of bounds have never been established up to now.

6. Comparing our Lie stability approach with Nekhoroshev procedure we observe the following:

- In Appendix 9 we determine the regions of quasi-convexity and directional quasi-convexity. They are represented in Fig. 9 in red and dark-blue, respectively. The union of both regions corresponds to the dark-blue region in Fig. 4, i.e. the region where there is Lie stability for any value of the frequencies (excluding the resonance lines).

- For the light-blue region, following the procedure based on Nekhoroshev theory we should proceed to the next step by checking a 3 -jet non- 
degeneracy condition, that is, $\mathcal{H}_{6}(\mathbf{I})=0$ implies $\mathbf{I}=\mathbf{0}$ (together with $\left.H_{2}(\mathbf{I})=\mathcal{H}_{4}(\mathbf{I})=0\right)$. When it occurs, $\mathcal{H}^{6}$ is steep at $\mathbf{I}=\mathbf{0}$ and estimates of Nekhoroshev type are available, see [5].

Applying the Lie stability approach we have obtained Lie stability excepting for a measure-zero set in the light-blue region by using the information given by $\mathcal{H}^{4}$. Thus, we improve largely the set of points in the plane of parameters $(C, A)$ where Nekhoroshev stability is attained. For the points where Theorem 4 does not provide information we can apply Nekhoroshev's point of view. In this sense, both procedures are complementary.

- On the one hand, we have bounded the solutions for exponentially long times by using Nekhoroshev estimates in the regions of quasi-convexity and directional quasi-convexity, see details in Appendix 9.

On the other hand, we have applied Corollary 1 to bound the solutions in the Lie stable cases. In Table 4 we show, for each case, the bound for the solution by applying Corollary 1 (first and second rows) and by applying Nekhoroshev estimates. The third column in the table shows the time-validity following both approaches. We highlight in boldface the best bounds in each case. The optimal case corresponds to the Lie stable cases when $\operatorname{dim} S=2$. In this situation Corollary 1 can be applied under no Diophantine conditions.

\begin{tabular}{|l|l|l|}
\hline Case & $|\mathbf{I}|$ & $|t|$ \\
\hline & $\epsilon^{\mathbf{1}}$ & $\exp \left(\epsilon^{-\mathbf{1} / \mathbf{2}}\right)$ \\
$\left(a_{1}\right) \bar{\omega}_{1}, \bar{\omega}_{2} \in \mathbb{Q}$ & $\epsilon^{1 / 3}$ & $\exp \left(\epsilon^{-1 / 3}\right)$ \\
& $\epsilon^{1 / 2}$ & $\exp \left(\epsilon^{-1 / 6}\right)$ \\
\hline$\left(a_{2}\right) \bar{\omega}_{1} \in \mathbb{Q}, \bar{\omega}_{2} \in \mathbb{R} \backslash \mathbb{Q}$ & $\epsilon^{\mathbf{1}}$ & $\exp \left(\epsilon^{-1 / 4}\right)$ \\
$\left(b_{2}\right) \bar{\omega}_{1}, \bar{\omega}_{2} \in \mathbb{R} \backslash \mathbb{Q} \& \exists \mathbf{k} \notin K$ & $\epsilon^{1 / 3}$ & $\exp \left(\epsilon^{-\mathbf{1} / \mathbf{3}}\right)$ \\
\hline & $\epsilon^{1 / 2}$ & $\exp \left(\epsilon^{-1 / 6}\right)$ \\
$\left(b_{1}\right) \bar{\omega}_{1}, \bar{\omega}_{2} \in \mathbb{R} \backslash \mathbb{Q} \& \exists \mathbf{k}$ & $\epsilon^{\mathbf{1}}$ & $\exp \left(\epsilon^{-1 / 6}\right)$ \\
& $\epsilon^{1 / 3}$ & $\exp \left(\epsilon^{-\mathbf{1} / 3}\right)$ \\
$\left(b_{2}\right) \bar{\omega}_{1}, \bar{\omega}_{2} \in \mathbb{R} \backslash \mathbb{Q} \& \exists \mathbf{k} \in K$ & $\epsilon^{1 / 2}$ & $\exp \left(\epsilon^{-1 / 6}\right)$ \\
$\left(b_{3}\right) \bar{\omega}_{1} \in \mathbb{R} \backslash \mathbb{Q}, \bar{\omega}_{2} \in \mathbb{Q}$ & $\epsilon^{\mathbf{1}}$ & $\exp \left(\epsilon^{-1 / 4}\right)$ \\
& $\epsilon^{1 / 3}$ & $\exp \left(\epsilon^{-\mathbf{1} / \mathbf{3}}\right)$ \\
\hline
\end{tabular}

Table 4 Estimates comparison. The second column indicates the bounds for the solution, whereas the third one states the time validity. For each case, the first row corresponds to the results obtained by the application of Corollary 1 in this paper and the remaining rows account for Nekhoroshev estimates. The best results in each case are shown in boldface type.

- A drawback of Theorem 5 and Corollary 1 is that, when there are more than one independent formal integral, i.e. $d>1$ a Diophantine condition for the frequency vector $\sigma$ is required. Fortunately, for a fixed $\nu$ the 
Lebesgue measure of the set of vectors in $\mathbb{R}^{d}$ that are not Diophantine for any $c>0$ is null.

Finally we stress that the calculations have been performed with MATHEMATICA, version 12 .

\section{Appendix: On Lie stabiliy}

In this appendix we review the main concepts and results related to Lie stability that are applied in Sects. 3 and 4.

Definition 1 Hamiltonian (3) with associated $\mathrm{H}_{2}$ as defined in (6) presents a resonance relation if there exists an integer vector $\mathbf{k}=\left(k_{1}, k_{2}, k_{3}\right) \neq \mathbf{0}$ such that

$$
k_{1} \omega_{1}-k_{2} \omega_{2}+k_{3} \omega_{3}=0 .
$$

The 1-norm of vector $\mathbf{k},|\mathbf{k}|_{1}=\left|k_{1}\right|+\left|k_{2}\right|+\left|k_{3}\right|$, is called the order of the resonance, while $\mathbf{k}$ is known as the resonance vector and $\omega=\left(\omega_{1},-\omega_{2}, \omega_{3}\right)$ stands for the frequency vector. The symbol $|\cdot|$ means the Euclidean norm.

Suppose $\left\{\mathbf{k}^{1}, \ldots, \mathbf{k}^{s}\right\}$ is a basis of the $\mathbb{Z}$-module $M_{\omega}$ associated to the possible resonances of $H_{2}(\mathbf{I})$ in $(6)$, where $0 \leq s \leq 2$. Specifically, $M_{\omega}$ is defined as

$$
M_{\omega}=\left\{\mathbf{k}=\left(k_{1}, k_{2}, k_{3}\right) \in \mathbb{Z}^{3} \mid k_{1} \omega_{1}-k_{2} \omega_{2}+k_{3} \omega_{3}=0\right\},
$$

and as it is finitely generated we also write

$$
M_{\omega}=\mathbf{k}^{1} \mathbb{Z}+\cdots+\mathbf{k}^{s} \mathbb{Z}=\left\{i_{1} \mathbf{k}^{1}+\cdots+i_{s} \mathbf{k}^{s} \mid i_{1}, \ldots, i_{s} \in \mathbb{Z}\right\},
$$

with the $\mathbf{k}^{j}$ linearly independent.

It is clear that $M_{\omega}=\{\mathbf{0}\}$ is equivalent to consider $\omega_{1}, \omega_{2}, \omega_{3}$ linearly independent over $\mathbb{Q}$, that is, $M_{\omega}=\{\mathbf{0}\}$ if and only if Hamiltonian (3) does not possess resonances.

Definition 2 Assume that $M_{\omega} \neq\{\mathbf{0}\}$. If $M_{\omega}$ is cyclic $(s=1)$ we say that the Hamiltonian (3) possesses a single resonance, otherwise $(s>1)$ we say that the system possesses multiple resonances.

The null space of $M_{\omega}$ is a vector subspace of $\mathbb{R}^{3}$ spanned by the vectors $\left\{\mathbf{a}_{1}, \ldots, \mathbf{a}_{d}\right\}$ with $d=3-s$ that satisfy $\mathbf{a}_{i} \cdot \mathbf{k}^{j}=0$, see details in [14], [15]. Setting $F_{l}=\mathbf{a}_{l} \cdot \mathbf{I}$, with $l=1, \ldots, d, \mathbf{I} \in \mathbb{R}^{3}$, we get the independent (formal) first integrals of the normal form Hamiltonian (10).

Consider the set

$$
S=\left\{\mathbf{I}=\left(I_{1}, I_{2}, I_{3}\right) \in \mathbb{R}^{3} \mid I_{j} \geq 0, \quad F_{1}(\mathbf{I})=\ldots=F_{d}(\mathbf{I})=0\right\},
$$

which was first introduced in [14]. It is the linear subspace of $\mathbb{R}^{3}$ that contains the essential vectors where the Hamiltonian is evaluated to decide on the Lie stability of the system. We note that $0 \leq \operatorname{dim} S \leq s$. 
The quadratic part of $H$ in terms of the formal first integrals $F_{k}$ assumes the form

$$
H_{2}(\mathbf{I})=\sum_{k=1}^{d} \sigma_{k} F_{k}(\mathbf{I}),
$$

where $\sigma_{k}$ are linear combinations of $\omega_{j}$, see for instance [16].

Definition 3 It is said that vector $\sigma=\left(\sigma_{1}, \ldots, \sigma_{d}\right)$ satisfies a Diophantine condition when there are fixed constants $c>0$ and $\nu>d-1$ such that

$$
\forall \mathbf{k} \in \mathbb{Z}^{d} \backslash\{\mathbf{0}\}, \quad|\mathbf{k} \cdot \sigma| \geq c|\mathbf{k}|_{1}^{-\nu} .
$$

As we deal with the different types of stability, it is convenient to recall them in the setting of the paper.

Definition 4 We say that the origin of $\mathbb{R}^{6}$ related to (3) is positively (respectively negatively) stable, if for every $\varepsilon>0$ there is $\delta>0$ such that if $\psi(t, \zeta)$ is the general solution of the system associated to $(3)$ then $|\psi(t, \zeta)|<\varepsilon$ for all $t \geq 0$ (respectively $t \leq 0$ ) whenever $|\zeta|<\delta$. The origin of $\mathbb{R}^{6}$ is said to be Liapunov stable if it is both positively and negatively stable.

Definition 5 We say that the origin of $\mathbb{R}^{6}$ related to (3) is formally stable if there exists a real formal power series $G(\mathbf{x})$, possibly divergent, which is an integral of $H$ in the formal sense and is positive definite near $\mathbf{x}=\mathbf{0}$.

Definition 6 We say that the origin of $\mathbb{R}^{6}$ related to (3) is Lie stable if there exists $p \geq 2$ such that the truncated Hamiltonian system in normal form associated to $\mathcal{H}^{q}$ is stable in the sense of Liapunov for any arbitrary $q \geq p$. (The truncated Hamiltonian system associated to $\mathcal{H}^{q}$ corresponds to equation (7) where $p$ is replaced by $q$.)

Remark 11 Lie stability is a type of formal stability, see for instance reference [15].

We state the main result about Lie stability given in [6] and [7]. Assume that Hamiltonian (3) is in normal form up to a high-enough order $p$ as is given in $(7)$ or $(8)$.

Theorem 4 Consider Hamiltonian (8) related to Hamiltonian (3) with $\mathbf{I} \in$ $S \backslash\{\mathbf{0}\}, \phi \in \mathbb{T}^{s}$.

(A) Suppose there is an even integer $j$ (with $4 \leq j \leq p$ ) such that $\mathcal{H}^{j}(\mathbf{I}, \phi) \neq$ 0 for $|\mathbf{I}|$ small enough and all $\phi$. Then the origin of $\mathbb{R}^{6}$ is Lie stable for the Hamiltonian system (3).

(B) Suppose there is an integer $i \geq 3$ such that $\mathcal{H}^{i}(\mathbf{I}, \phi)$ changes sign for some $\mathbf{I}, \phi$. Then there is no index $j$ (with $i<j \leq p$ ) such that $\mathcal{H}^{j}(\mathbf{I}, \phi) \neq 0$ for $|\mathbf{I}|$ sufficiently small. 
Remark 12 When $S=\{\mathbf{0}\}$ Lie stability always holds, details can be looked up in [15], [7].

Assume that $\mathbf{x}\left(t, \mathbf{x}_{0}\right)$ is a solution of the Hamiltonian system associated to $H$ in (3) with initial condition $\mathbf{x}_{0}$. For the Lie stable equilibria we give an estimate of the solution's evolution according to the following theorem, see more details in [6], [7].

Theorem 5 If the real analytic Hamiltonian (3) has the origin of $\mathbb{R}^{6}$ as a formally stable equilibrium in accordance to hypotheses (A) of Theorem 4, while the frequency vector $\sigma$ satisfies the Diophantine condition (24), then there exist $C>0, E>0, a>1$ and $\varepsilon_{0}>0$ such that for all $\varepsilon \in\left(0, \varepsilon_{0}\right)$, and for all $\mathbf{x}_{0}$ with $\left|\mathbf{x}_{0}\right|<\varepsilon$ we have

$$
\left|\mathbf{x}\left(t, \mathbf{x}_{0}\right)\right|<a \varepsilon^{2 / j} \quad \text { for all } t \text { with } \quad 0 \leq t \leq T=C \exp \left(\frac{E}{\varepsilon^{1 /(\nu+1)}}\right) .
$$

The proofs of Theorems 4 and 5 are given in [6], [7].

We can improve the confinement's bound on the solution with a slight change in hypotheses of Theorem 4 part (A), as follows.

Corollary 1 Suppose there are two even integers $j^{\prime}, j$ such that $4 \leq j^{\prime} \leq j \leq$ $p, \mathcal{H}_{k}(\mathbf{x})=0$ for $k=3, \ldots, j^{\prime}-1$ and $\sum_{k=j^{\prime}}^{j} \mathcal{H}_{k}(\mathbf{x}) \neq 0$ for all $\mathbf{x}$ associated to $\mathbf{I} \in S \backslash\{\mathbf{0}\}$. Suppose in addition that the frequency vector $\sigma$ is Diophantine. Then with the same notation as in Theorem 5, the estimates are of the form

$$
\left|\mathbf{x}\left(t, \mathbf{x}_{0}\right)\right|<a \varepsilon^{j^{\prime} / j} \quad \text { for all } t \text { with } \quad 0 \leq t \leq T=C \exp \left(\frac{K}{\varepsilon^{1 /(\nu+1)}}\right) .
$$

See the proof and associated examples in [7].

Other recent works on Lie stable and unstable systems are due to dos Santos and coworkers $[14,15]$ where the authors establish several criteria dealing with Lie stable equilibria in cases of single and multiple resonances. We present the results for $n=3$.

Let $\mathbf{k}=\left(k_{1}, k_{2}, k_{3}\right)$ be a vector of resonance. In the case $k_{1}, k_{2}, k_{3} \geq 0$ or $\leq 0$ and without loss of generality, we assume $k_{1} \neq 0$. We define the function

$$
\begin{aligned}
F_{m}\left(I_{1}, \phi\right)= & \mathcal{H}^{m}\left(\frac{k_{1}}{k_{1}} I_{1}, \frac{k_{2}}{k_{1}} I_{1}, \frac{k_{3}}{k_{1}} I_{1}, \phi\right) \\
= & A_{2} I_{1}+\ldots+A_{2 l} I_{1}^{l}+\Psi_{|\mathbf{k}|}(\phi) I_{1}^{|\mathbf{k}| / 2}+\Psi_{|\mathbf{k}|+1}(\phi) I_{1}^{(|\mathbf{k}|+1) / 2} \\
& +\ldots+\Psi_{m}(\phi) I_{1}^{m / 2}
\end{aligned}
$$

where $\phi=\mathbf{k}^{1} \cdot \theta=k_{1}^{1} \theta_{1}+k_{2}^{1} \theta_{2}+k_{3}^{1} \theta_{3}$ is the resonant angle and $2 l$ is an even natural number smaller than or equal to $|\mathbf{k}|$ and

$$
A_{2 j}=k_{1}^{-j} \mathcal{H}_{2 j}(\mathbf{k}), j=1, \ldots, l \text { and } \Psi_{s}(\phi)=k_{1}^{-s / 2} \mathcal{H}_{s}(\mathbf{k}, \phi),
$$

where $s=|\mathbf{k}|, \ldots, m$. The main result for single resonances where the linear part is diagonalisable is as follows. 
Theorem 6 Assume that the system (3) possesses a single resonance, with the vector of resonance given by $\mathbf{k}=\left(k_{1}, k_{2}, k_{3}\right)$.

(A) If there exist $i, j \in\{1,2,3\}, i \neq j$, such that $k_{i} k_{j}<0$, then the null solution of (3) is Lie stable.

(B) If $k_{1}, k_{2}, k_{3} \geq 0$ or $\leq 0$ and one of the coefficients $A_{2 j} \neq 0$ for some $j=1, \ldots,|\mathbf{k}|-1$ or when $A_{2 j}=0$ for all $j=1, \ldots,|\mathbf{k}|-1$ but the function in (25) $\Psi_{|\mathbf{k}|}(\phi) \neq 0$ for all $\phi$, then the null solution of (3) is Lie stable.

(C) If $k_{1}, k_{2}, k_{3} \geq 0$ or $\leq 0$ and there exists $\phi=\phi^{*}$ such that $\Psi_{|\mathbf{k}|}\left(\phi^{*}\right)=0$ and $\Psi_{|\mathbf{k}|}^{\prime}\left(\phi^{*}\right) \neq 0$ (i.e., $\phi^{*}$ is a simple zero), then the null solution of (3) is unstable.

Now, we assume that $\left|\mathbf{k}^{1}\right|<\left|\mathbf{k}^{2}\right| \leq \ldots \leq\left|\mathbf{k}^{s}\right|$ and $2\left|\mathbf{k}^{1}\right|-2<\left|\mathbf{k}^{2}\right|$. If $\eta=\left|\mathbf{k}^{1}\right|$, the function $\mathcal{H}^{2 \eta-2}$ takes the form

$$
\mathcal{H}^{2 \eta-2}=H_{2}(\mathbf{I})+\ldots+\mathcal{H}_{2 l}(\mathbf{I})+\mathcal{H}_{\eta}\left(\mathbf{I}, \mathbf{k}^{1} \cdot \theta\right)+\ldots+\mathcal{H}_{2 \eta-2}\left(\mathbf{I}, \mathbf{k}^{1} \cdot \theta\right)
$$

where $2 l$ is an even natural number smaller than $\eta$. We suppose that $H$ is in normal form up to orden $2 \eta-2$. In the case $k_{1}^{1}, k_{2}^{1}, k_{3}^{1} \geq 0$ with $k_{1}^{1}>0$, we consider the auxiliary function

$$
\Psi(\phi)=\left(k_{1}^{1}\right)^{-\eta / 2} \mathcal{H}_{\eta}\left(\mathbf{k}^{1}, \phi\right)
$$

Theorem 7 With the same notations as above, if $k_{i}^{1} \geq 0, k_{1}^{1}>0, \mathcal{H}_{4}\left(\mathbf{k}^{1}\right)=$ $\ldots=\mathcal{H}_{2 l}\left(\mathbf{k}^{1}\right)=0$ and if there exists $\phi^{*}$ such that $\Psi\left(\phi^{*}\right)=0$ and $\Psi^{\prime}\left(\phi^{*}\right) \neq 0$, then the null solution of (3) is unstable.

\section{Appendix: Coefficients lists}

We include the coefficients of some formulae that appear in this article.

Table 5 contains the polynomials' coefficients of the Sturm sequence associated to the characteristic polynomial $p_{B}$ corresponding to the linearisation matrix related to $P_{j}$, with $j=1, \ldots, 4$. 


\begin{tabular}{|c|c|}
\hline$b_{0}$ & $\begin{aligned} \frac{1}{9 A^{2} C^{2}} & \left(-27 A^{5}(C+1)+9 A^{4}\left(8 C^{2}+9 C+4\right)-3 A^{3}\left(21 C^{3}-C^{2}+59 C-6\right)\right. \\
& +2 A^{2}\left(9 C^{4}-24 C^{3}+22 C^{2}+51 C-16\right) \\
& \left.+A\left(-9 C^{4}+93 C^{3}-111 C^{2}+26 C+1\right)+(C-1)^{2}(3 C+4)\right)\end{aligned}$ \\
\hline$b_{1}$ & $\begin{aligned} \frac{2}{9 A^{2} C^{2}} & \left(9 A^{4}\left(C^{2}-C+1\right)-3 A^{3}\left(6 C^{3}-5 C^{2}+2 C+4\right)\right. \\
& +A^{2}\left(9 C^{4}-6 C^{3}-17 C^{2}+29 C-2\right)+A\left(3 C^{3}+5 C^{2}-12 C+4\right) \\
& \left.+(C-1)^{2}\right)\end{aligned}$ \\
\hline$\alpha_{1}$ & $9 A^{4}-12 A^{3}(C+1)-2 A^{2}\left(6 C^{2}-15 C+1\right)+4 A\left(3 C^{2}-4 C+1\right)+(C-1)^{2}$ \\
\hline$\alpha_{2}$ & $9 A^{3}(C-1)+A^{2}\left(-18 C^{2}+15 C+6\right)+A\left(9 C^{3}-6 C^{2}-5 C-1\right)+3 C^{2}-7 C+4$ \\
\hline$\alpha_{3}$ & $\begin{array}{l}9 A^{4}\left(C^{2}-C+1\right)-3 A^{3}\left(6 C^{3}-5 C^{2}+2 C+4\right) \\
+A^{2}\left(9 C^{4}-6 C^{3}-17 C^{2}+29 C-2\right)+A\left(3 C^{3}+5 C^{2}-12 C+4\right)+(C-1)^{2}\end{array}$ \\
\hline
\end{tabular}

Table 5 Coefficients of the polynomials $p_{2}(t)$ and $p_{3}(t)$ corresponding to the Sturm sequence associated to $p_{B}$.

Tables 6 and 7 account for the terms that define the normalisation of $H_{4}$ in the absence of resonances of orders 3 and 4 . 


\begin{tabular}{|c|c|c|c|}
\hline$f_{1}$ & $\omega_{1}^{2}-1$ & $g_{1}$ & $\omega_{2}^{2}-1$ \\
\hline$f_{2}$ & $\omega_{1}^{2}-4$ & $g_{2}$ & $\omega_{2}^{2}-4$ \\
\hline$f_{3}$ & $\omega_{1}^{4}+14 \omega_{1}^{2}-8$ & $g_{3}$ & $\omega_{2}^{4}+14 \omega_{2}^{2}-8$ \\
\hline$f_{4}$ & $2 \omega_{1}^{4}-3 \omega_{1}^{2}+4$ & $g_{4}$ & $2 \omega_{2}^{4}-3 \omega_{2}^{2}+4$ \\
\hline$f_{5}$ & $-9 \omega_{1}^{8}+26 \omega_{1}^{6}+12 \omega_{1}^{4}-48 \omega_{1}^{2}+64$ & $g_{5}$ & $-9 \omega_{2}^{8}+26 \omega_{2}^{6}+12 \omega_{2}^{4}-48 \omega_{2}^{2}+64$ \\
\hline$f_{6}$ & $15 \omega_{1}^{8}+5 \omega_{1}^{6}-108 \omega_{1}^{4}+132 \omega_{1}^{2}-80$ & $g_{6}$ & $15 \omega_{2}^{8}+5 \omega_{2}^{6}-108 \omega_{2}^{4}+132 \omega_{2}^{2}-80$ \\
\hline$f_{7}$ & $5 \omega_{1}^{2}-4$ & $g_{7}$ & $5 \omega_{2}^{2}-4$ \\
\hline$f_{8}$ & $3 \omega_{1}^{4}-4 \omega_{1}^{2}+4$ & $g_{8}$ & $3 \omega_{2}^{4}-4 \omega_{2}^{2}+4$ \\
\hline$f_{9}$ & $-7 \omega_{1}^{6}+24 \omega_{1}^{4}-24 \omega_{1}^{2}+16$ & $g_{9}$ & $-7 \omega_{2}^{6}+24 \omega_{2}^{4}-24 \omega_{2}^{2}+16$ \\
\hline$f_{10}$ & $4 \omega_{1}^{2}-\omega_{3}^{2}$ & $g_{10}$ & $4 \omega_{2}^{2}-\omega_{3}^{2}$ \\
\hline$h_{1}$ & $\omega_{1}^{2}-\omega_{2}^{2}$ & $h_{2}$ & $\omega_{1}^{2} \omega_{2}^{2}-4$ \\
\hline$h_{3}$ & \multicolumn{3}{|l|}{$\omega_{1}^{4}-2\left(\omega_{2}^{2}+\omega_{3}^{2}\right) \omega_{1}^{2}+\left(\omega_{2}^{2}-\omega_{3}^{2}\right)^{2}$} \\
\hline$t_{1}$ & \multicolumn{3}{|c|}{$\begin{array}{l}\omega_{2}^{12}+\left(6-4 \omega_{3}^{2}\right) \omega_{2}^{10}+\left(3 \omega_{3}^{4}+60 \omega_{3}^{2}-88\right) \omega_{2}^{8}-2\left(8 \omega_{3}^{4}+97 \omega_{3}^{2}-45\right) \omega_{2}^{6} \\
+2\left(11 \omega_{3}^{4}+78 \omega_{3}^{2}-20\right) \omega_{2}^{4}+24\left(3 \omega_{3}^{2}+16\right) \omega_{2}^{2}-128\end{array}$} \\
\hline$t_{2}$ & \multicolumn{3}{|c|}{$\begin{array}{l}3 \omega_{2}^{12}-\left(11 \omega_{3}^{2}+19\right) \omega_{2}^{10}+\left(8 \omega_{3}^{4}+97 \omega_{3}^{2}-45\right) \omega_{2}^{8}-2\left(17 \omega_{3}^{4}+46 \omega_{3}^{2}+28\right) \omega_{2}^{6} \\
+\left(25 \omega_{3}^{4}-280 \omega_{3}^{2}+192\right) \omega_{2}^{4}+4\left(7 \omega_{3}^{4}+117 \omega_{3}^{2}+65\right) \omega_{2}^{2}-128\left(\omega_{3}^{2}+1\right)\end{array}$} \\
\hline$t_{3}$ & \multicolumn{3}{|c|}{$\begin{array}{l}3 \omega_{2}^{12}-7\left(2 \omega_{3}^{2}+7\right) \omega_{2}^{10}+\left(11 \omega_{3}^{4}+78 \omega_{3}^{2}-20\right) \omega_{2}^{8}+\left(-25 \omega_{3}^{4}+280 \omega_{3}^{2}-192\right) \omega_{2}^{6} \\
-8\left(10 \omega_{3}^{4}+103 \omega_{3}^{2}-81\right) \omega_{2}^{4}+4\left(53 \omega_{3}^{4}+177 \omega_{3}^{2}-32\right) \omega_{2}^{2}-64\left(\omega_{3}^{4}+3 \omega_{3}^{2}+1\right)\end{array}$} \\
\hline$t_{4}$ & \multicolumn{3}{|c|}{$\begin{array}{l}\omega_{2}^{12}-\left(\omega_{3}^{2}+2\right) \omega_{2}^{10}+\left(9 \omega_{3}^{2}+48\right) \omega_{2}^{8}-\left(7 \omega_{3}^{4}+117 \omega_{3}^{2}+65\right) \omega_{2}^{6} \\
+\left(53 \omega_{3}^{4}+177 \omega_{3}^{2}-32\right) \omega_{2}^{4}-16\left(5 \omega_{3}^{4}+3 \omega_{3}^{2}-2\right) \omega_{2}^{2}+16\left(\omega_{3}^{4}+\omega_{3}^{2}\right) \\
\end{array}$} \\
\hline$t_{5}$ & \multicolumn{3}{|c|}{$\omega_{2}^{8}+\left(2 \omega_{3}^{2}-3\right) \omega_{2}^{6}-\left(11 \omega_{3}^{2}+19\right) \omega_{2}^{4}+7\left(2 \omega_{3}^{2}+7\right) \omega_{2}^{2}+4\left(\omega_{3}^{2}+2\right)$} \\
\hline$t_{6}$ & \multicolumn{3}{|c|}{$\begin{array}{l}\left(37 \omega_{3}^{4}+293 \omega_{3}^{2}+48\right) \omega_{2}^{10}-2\left(23 \omega_{3}^{6}+271 \omega_{3}^{4}-14 \omega_{3}^{2}+368\right) \omega_{2}^{8} \\
+\left(9 \omega_{3}^{8}+361 \omega_{3}^{6}+608 \omega_{3}^{4}-3088 \omega_{3}^{2}-768\right) \omega_{2}^{6} \\
+4 \omega_{3}^{2}\left(-11 \omega_{3}^{6}-106 \omega_{3}^{4}+843 \omega_{3}^{2}+391\right) \omega_{2}^{4}+16\left(\omega_{3}^{8}-60 \omega_{3}^{6}-399 \omega_{3}^{4}\right. \\
\left.+74 \omega_{3}^{2}+16\right) \omega_{2}^{2}+64\left(\omega_{3}^{8}+16 \omega_{3}^{6}+38 \omega_{3}^{4}+10 \omega_{3}^{2}+12\right)\end{array}$} \\
\hline$t_{7}$ & \multicolumn{3}{|c|}{$\begin{array}{l}2\left(5 \omega_{3}^{4}+72 \omega_{3}^{2}+18\right) \omega_{2}^{10}-\left(11 \omega_{3}^{6}+247 \omega_{3}^{4}+478 \omega_{3}^{2}+96\right) \omega_{2}^{8} \\
+\left(\omega_{3}^{8}+119 \omega_{3}^{6}+1054 \omega_{3}^{4}-592 \omega_{3}^{2}-128\right) \omega_{2}^{6} \\
-4\left(2 \omega_{3}^{8}+86 \omega_{3}^{6}+157 \omega_{3}^{4}-516 \omega_{3}^{2}+336\right) \omega_{2}^{4} \\
+16\left(\omega_{3}^{8}+6 \omega_{3}^{6}-122 \omega_{3}^{4}-113 \omega_{3}^{2}-72\right) \omega_{2}^{2}+64\left(5 \omega_{3}^{6}+17 \omega_{3}^{4}+13 \omega_{3}^{2}+20\right)\end{array}$} \\
\hline$t_{8}$ & \multicolumn{3}{|c|}{$\begin{array}{l}2\left(9 \omega_{3}^{4}+35 \omega_{3}^{2}-40\right) \omega_{2}^{10}-\left(27 \omega_{3}^{6}+115 \omega_{3}^{4}-268 \omega_{3}^{2}+336\right) \omega_{2}^{8} \\
+\left(9 \omega_{3}^{8}+121 \omega_{3}^{6}-469 \omega_{3}^{4}-865 \omega_{3}^{2}+864\right) \omega_{2}^{6} \\
+\left(-24 \omega_{3}^{8}+161 \omega_{3}^{6}+1525 \omega_{3}^{4}+752 \omega_{3}^{2}-832\right) \omega_{2}^{4} \\
-4\left(6 \omega_{3}^{8}+199 \omega_{3}^{6}+376 \omega_{3}^{4}-41 \omega_{3}^{2}-96\right) \omega_{2}^{2}+16 \omega_{3}^{2}\left(3 \omega_{3}^{6}+31 \omega_{3}^{4}+38 \omega_{3}^{2}-26\right)\end{array}$} \\
\hline
\end{tabular}

Table 6 Coefficients of Hamiltonian function (11). Part I. 


\begin{tabular}{|c|c|c|c|}
\hline$t_{9}$ & \multicolumn{3}{|c|}{$\begin{array}{l}\left(\omega_{3}^{4}+34 \omega_{3}^{2}+4\right) \omega_{2}^{8}-\left(\omega_{3}^{6}+39 \omega_{3}^{4}+127 \omega_{3}^{2}-40\right) \omega_{2}^{6} \\
+\left(10 \omega_{3}^{6}+163 \omega_{3}^{4}+133 \omega_{3}^{2}+16\right) \omega_{2}^{4} \\
+4\left(-6 \omega_{3}^{6}-29 \omega_{3}^{4}+12 \omega_{3}^{2}+120\right) \omega_{2}^{2}+16 \omega_{3}^{2}\left(8-3 \omega_{3}^{2}\right)\end{array}$} \\
\hline$t_{10}$ & \multicolumn{3}{|c|}{$\begin{array}{l}\omega_{2}^{2}\left(-2 \omega_{2}^{4}+17 \omega_{2}^{2}-36\right) \omega_{3}^{4}+\left(\omega_{2}^{2}-4\right)^{2}\left(3 \omega_{2}^{4}-6 \omega_{2}^{2}+5\right) \omega_{3}^{2} \\
+4\left(2 \omega_{2}^{6}-7 \omega_{2}^{4}+20 \omega_{2}^{2}+48\right)\end{array}$} \\
\hline$t_{11}$ & \multicolumn{3}{|c|}{$\begin{array}{l}\left(4 \omega_{2}^{4}+5 \omega_{2}^{2}-24\right) \omega_{3}^{8}-\left(9 \omega_{2}^{6}+18 \omega_{2}^{4}-130 \omega_{2}^{2}+112\right) \omega_{3}^{6} \\
+\left(5 \omega_{2}^{8}+16 \omega_{2}^{6}-191 \omega_{2}^{4}+293 \omega_{2}^{2}-72\right) \omega_{3}^{4} \\
+\left(-3 \omega_{2}^{8}+117 \omega_{2}^{6}-181 \omega_{2}^{4}-40 \omega_{2}^{2}+80\right) \omega_{3}^{2}-48 \omega_{2}^{4}\left(\omega_{2}^{2}-1\right)^{2}\end{array}$} \\
\hline$t_{12}$ & \multicolumn{3}{|c|}{$\begin{array}{l}3 \omega_{3}^{2} \omega_{2}^{8}-\left(\omega_{3}^{4}+10 \omega_{3}^{2}+4\right) \omega_{2}^{6}+2\left(\omega_{3}^{4}+8 \omega_{3}^{2}+2\right) \omega_{2}^{4} \\
+\left(-5 \omega_{3}^{4}-5 \omega_{3}^{2}+48\right) \omega_{2}^{2}-16 \omega_{3}^{2}\end{array}$} \\
\hline$t_{13}$ & \multirow{3}{*}{\multicolumn{3}{|c|}{$\begin{array}{l}\omega_{3}^{2} \omega_{2}^{12}-2\left(\omega_{3}^{4}+19 \omega_{3}^{2}-4\right) \omega_{2}^{10}+\left(\omega_{3}^{6}+35 \omega_{3}^{4}+111 \omega_{3}^{2}+8\right) \omega_{2}^{8} \\
-\left(7 \omega_{3}^{6}+107 \omega_{3}^{4}+34 \omega_{3}^{2}+64\right) \omega_{2}^{6}+2\left(9 \omega_{3}^{6}+57 \omega_{3}^{4}-82 \omega_{3}^{2}+240\right) \omega_{2}^{4} \\
-4\left(9 \omega_{3}^{6}+\omega_{3}^{4}-104 \omega_{3}^{2}-144\right) \omega_{2}^{2}-64 \omega_{3}^{2}\left(3 \omega_{3}^{2}+4\right) \\
12\left(\omega_{3}^{2}+1\right) \omega_{2}^{14}+\left(-26 \omega_{3}^{4}-135 \omega_{3}^{2}+48\right) \omega_{2}^{12} \\
+\left(16 \omega_{3}^{6}+192 \omega_{3}^{4}+121 \omega_{3}^{2}-104\right) \omega_{2}^{10} \\
-\left(2 \omega_{3}^{8}+86 \omega_{3}^{6}+157 \omega_{3}^{4}-516 \omega_{3}^{2}+336\right) \omega_{2}^{8} \\
+\omega_{3}^{2}\left(11 \omega_{3}^{6}+106 \omega_{3}^{4}-843 \omega_{3}^{2}-391\right) \omega_{2}^{6} \\
+\left(-24 \omega_{3}^{8}+161 \omega_{3}^{6}+1525 \omega_{3}^{4}+752 \omega_{3}^{2}-832\right) \omega_{2}^{4} \\
-4\left(\omega_{3}^{8}+148 \omega_{3}^{6}+484 \omega_{3}^{4}+141 \omega_{3}^{2}+48\right) \omega_{2}^{2}+64 \omega_{3}^{2}\left(\omega_{3}^{6}+8 \omega_{3}^{4}+12 \omega_{3}^{2}+4\right) \\
-4\left(3 \omega_{3}^{2}+1\right) \omega_{2}^{14}+\left(25 \omega_{3}^{4}+221 \omega_{3}^{2}-60\right) \omega_{2}^{12} \\
-\left(14 \omega_{3}^{6}+319 \omega_{3}^{4}+641 \omega_{3}^{2}-144\right) \omega_{2}^{10} \\
+\left(\omega_{3}^{8}+119 \omega_{3}^{6}+1054 \omega_{3}^{4}-592 \omega_{3}^{2}-128\right) \omega_{2}^{8} \\
+\left(-9 \omega_{3}^{8}-361 \omega_{3}^{6}-608 \omega_{3}^{4}+3088 \omega_{3}^{2}+768\right) \omega_{2}^{6} \\
+4\left(9 \omega_{3}^{8}+121 \omega_{3}^{6}-469 \omega_{3}^{4}-865 \omega_{3}^{2}+864\right) \omega_{2}^{4} \\
-16\left(4 \omega_{3}^{8}-9 \omega_{3}^{6}-207 \omega_{3}^{4}-298 \omega_{3}^{2}-144\right) \omega_{2}^{2}-256 \omega_{3}^{2}\left(3 \omega_{3}^{4}+10 \omega_{3}^{2}+6\right) \\
4\left(\omega_{3}^{2}+3\right) \omega_{2}^{14}+\left(-9 \omega_{3}^{4}-39 \omega_{3}^{2}+8\right) \omega_{2}^{12}+2\left(3 \omega_{3}^{6}+13 \omega_{3}^{4}-2 \omega_{3}^{2}-60\right) \omega_{2}^{10}\end{array}$}} \\
\hline$t_{14}$ & & & \\
\hline$t_{15}$ & & & \\
\hline$t_{16}$ & \multicolumn{3}{|c|}{$\begin{array}{l}+\left(-\omega_{3}^{8}-6 \omega_{3}^{6}+122 \omega_{3}^{4}+113 \omega_{3}^{2}+72\right) \omega_{2}^{8} \\
+\left(\omega_{3}^{8}-60 \omega_{3}^{6}-399 \omega_{3}^{4}+74 \omega_{3}^{2}+16\right) \omega_{2}^{6} \\
+\left(6 \omega_{3}^{8}+199 \omega_{3}^{6}+376 \omega_{3}^{4}-41 \omega_{3}^{2}-96\right) \omega_{2}^{4} \\
-\omega_{3}^{2}\left(29 \omega_{3}^{6}+242 \omega_{3}^{4}+365 \omega_{3}^{2}-120\right) \omega_{2}^{2}+16\left(2 \omega_{3}^{8}+7 \omega_{3}^{6}+6 \omega_{3}^{4}+\omega_{3}^{2}\right)\end{array}$} \\
\hline$t_{17}$ & \multicolumn{3}{|c|}{$\begin{array}{l}4 \omega_{2}^{12}-\left(5 \omega_{3}^{2}+8\right) \omega_{2}^{10}+\left(-3 \omega_{3}^{4}+3 \omega_{3}^{2}-8\right) \omega_{2}^{8} \\
+\left(5 \omega_{3}^{6}+14 \omega_{3}^{4}+16 \omega_{3}^{2}+12\right) \omega_{2}^{6}-\omega_{3}^{2}\left(\omega_{3}^{6}+11 \omega_{3}^{4}+24 \omega_{3}^{2}-6\right) \omega_{2}^{4} \\
+2 \omega_{3}^{2}\left(\omega_{3}^{6}+10 \omega_{3}^{4}+7 \omega_{3}^{2}-10\right) \omega_{2}^{2}-4 \omega_{3}^{4}\left(\omega_{3}^{2}+1\right)^{2}\end{array}$} \\
\hline$t_{18}$ & $\omega_{2}^{6}-6 \omega_{2}^{4}+6 \omega_{2}^{2}+8$ & & $\left(\omega_{2}^{2}-\omega_{3}^{2}\right)\left(\omega_{2}^{2}-\omega_{3}^{2}-1\right)$ \\
\hline$t_{20}$ & $\omega_{2}^{2} \omega_{3}^{2}-4$ & $t_{21}$ & $\left(\omega_{2}^{2}-\omega_{3}^{2}\right)\left(\omega_{2}^{2}-\omega_{3}^{2}-1\right)^{2}$ \\
\hline
\end{tabular}

Table 7 Coefficients of Hamiltonian function (11). Part II. 
In Tables 8, 9, 10 11, 12 and 13 we present the coefficients that define the normalisation of $H_{3}$ in the resonances related to the vectors $\mathbf{k}^{2}$, and $H_{4}$ in $\mathbf{k}^{11}$ and $\mathbf{k}^{12}$.

$$
\begin{array}{|l|l|}
\hline \gamma_{1} & \frac{-\omega_{1}^{2}\left(\omega_{2}^{4}+\omega_{2}^{2}+2\right)+2\left(\omega_{2}^{2}+1\right)}{4\left(\omega_{1}^{2}-\omega_{2}^{2}\right)} \sqrt{\frac{\left(4-\omega_{1}^{2}\right)\left(4-\omega_{2}^{2}\right)}{3 \omega_{2}\left(4-\omega_{1}^{2} \omega_{2}^{2}\right)}} \\
\hline
\end{array}
$$

Table 8 Resonance associated to $\mathbf{k}^{2}$ : Coefficient $\gamma_{1}$ in the normal form up to order 1 given in (17).

\begin{tabular}{|l|l|}
\hline$c_{200}$ & $\frac{\left(4-9 \omega_{2}^{2}\right) \sum_{i=1}^{9} A_{i} \omega_{2}^{18-2 i}}{12288 \omega_{2}^{4}\left(1-\omega_{2}^{2}\right)\left(4-9 \omega_{2}^{4}\right)\left(36 \omega_{2}^{2}-\omega_{3}^{2}\right)}$ \\
\hline$c_{020}$ & $\frac{\left(4-\omega_{2}^{2}\right) \sum_{i=1}^{9} B_{i} \omega_{2}^{18-2 i}}{12288 \omega_{2}^{4}\left(1-9 \omega_{2}^{2}\right)\left(4-9 \omega_{2}^{4}\right)\left(4 \omega_{2}^{2}-\omega_{3}^{2}\right)}$ \\
\hline$c_{002}$ & $-\frac{\left(4-\omega_{2}^{2}\right)\left(4-9 \omega_{2}^{2}\right)}{12\left(4-9 \omega_{2}^{4}\right)}$ \\
\hline$c_{110}$ & $\frac{\sum_{i=1}^{10} D_{i} \omega_{2}^{20-2 i}}{9216 \omega_{2}^{4}\left(4-9 \omega_{2}^{4}\right)\left(4 \omega_{2}^{2}-\omega_{3}^{2}\right)\left(16 \omega_{2}^{2}-\omega_{3}^{2}\right)}$ \\
\hline$c_{011}$ & $\frac{\sum_{i=1}^{11} E_{i} \omega_{2}^{22-2 i}}{192 \omega_{2}^{3} \omega_{3}\left(4-9 \omega_{2}^{4}\right)\left(9 \omega_{2}^{4}-40 \omega_{2}^{2}+4\right)\left(4 \omega_{2}^{2}-\omega_{3}^{2}\right)\left(16 \omega_{2}^{2}-\omega_{3}^{2}\right)}$ \\
\hline$c_{101}$ & $\frac{\sum_{i=1}^{12} F_{i} \omega_{2}^{24-2 i}}{576 \omega_{2}^{3} \omega_{3}\left(4-9 \omega_{2}^{4}\right)\left(9 \omega_{2}^{4}-40 \omega_{2}^{2}+4\right)\left(4 \omega_{2}^{2}-\omega_{3}^{2}\right)\left(16 \omega_{2}^{2}-\omega_{3}^{2}\right)\left(36 \omega_{2}^{2}-\omega_{3}^{2}\right)}$ \\
\hline$\gamma_{2}$ & $\frac{\sqrt{4-9 \omega_{2}^{2}} \sqrt{1-\omega_{2}^{2}} \sqrt{4-\omega_{2}^{2}} \sum_{i=1}^{7} T_{i} \omega_{2}^{14-2 i}}{3072 \sqrt{3} \omega_{2}^{4} \sqrt{1-9 \omega_{2}^{2}}\left(4-9 \omega_{2}^{4}\right)\left(4 \omega_{2}^{2}-\omega_{3}^{2}\right)}$ \\
\hline
\end{tabular}

Table 9 Resonance associated to $\mathbf{k}^{11}$. Coefficients of the normal form $\mathcal{H}_{4}$ given in (19). 


\begin{tabular}{|l|}
\hline$A_{1}=-52488$ \\
\hline$A_{2}=2187\left(\omega_{3}^{2}+144\right)$ \\
\hline$A_{3}=-81\left(151 \omega_{3}^{2}+6180\right)$ \\
\hline$A_{4}=36\left(437 \omega_{3}^{2}+10651\right)$ \\
\hline$A_{5}=-4\left(2169 \omega_{3}^{2}-44176\right)$ \\
\hline$A_{6}=-4\left(1415 \omega_{3}^{2}+58636\right)$ \\
\hline$A_{7}=16\left(383 \omega_{3}^{2}+1905\right)$ \\
\hline$A_{8}=-16\left(49 \omega_{3}^{2}+85\right)$ \\
\hline$A_{9}=64\left(\omega_{3}^{2}+1\right)$ \\
\hline$B_{1}=-52488$ \\
\hline$B_{2}=729\left(27 \omega_{3}^{2}+368\right)$ \\
\hline$B_{3}=-81\left(711 \omega_{3}^{2}+5860\right)$ \\
\hline$B_{4}=36\left(3933 \omega_{3}^{2}+2419\right)$ \\
\hline$B_{5}=-36\left(481 \omega_{3}^{2}-7824\right)$ \\
\hline$B_{6}=-4\left(15615 \omega_{3}^{2}+33004\right)$ \\
\hline$B_{7}=112\left(169 \omega_{3}^{2}+215\right)$ \\
\hline$B_{8}=-16\left(121 \omega_{3}^{2}+125\right)$ \\
\hline$B_{9}=64\left(\omega_{3}^{2}+1\right)$ \\
\hline$D_{1}=-419904$ \\
\hline$D_{2}=87480\left(3 \omega_{3}^{2}+32\right)$ \\
\hline$D_{3}=-243\left(81 \omega_{3}^{4}+7032 \omega_{3}^{2}+20096\right)$ \\
\hline$D_{4}=180\left(648 \omega_{3}^{4}+17055 \omega_{3}^{2}+4928\right)$ \\
\hline$D_{5}=-144\left(1359 \omega_{3}^{4}+4846 \omega_{3}^{2}-7840\right)$ \\
\hline$D_{6}=540\left(75 \omega_{3}^{4}-1716 \omega_{3}^{2}-4096\right)$ \\
\hline$D_{7}=16\left(3393 \omega_{3}^{4}+51516 \omega_{3}^{2}+69920\right)$ \\
\hline$D_{8}=-80\left(477 \omega_{3}^{4}+3929 \omega_{3}^{2}+2048\right)$ \\
\hline$D_{9}=128\left(127 \omega_{3}^{4}+273 \omega_{3}^{2}+64\right)$ \\
\hline$D_{10}=-1280 \omega_{3}^{2}\left(\omega_{3}^{2}+1\right)$ \\
\hline
\end{tabular}

Table 10 Resonance related to $\mathbf{k}^{11}$. Part II: Terms appearing in Table 9, that contain the coefficients of the normal form $\mathcal{H}_{4}$ given in (19). 


\begin{tabular}{|l|}
\hline$E_{1}=-45927 \omega_{3}^{2}$ \\
\hline$E_{2}=729\left(9 \omega_{3}^{4}+786 \omega_{3}^{2}+64\right)$ \\
\hline$E_{3}=-243\left(327 \omega_{3}^{4}+10645 \omega_{3}^{2}+1984\right)$ \\
\hline$E_{4}=9\left(81 \omega_{3}^{6}+40545 \omega_{3}^{4}+568528 \omega_{3}^{2}+92928\right)$ \\
\hline$E_{5}=-7209 \omega_{3}^{6}-880677 \omega_{3}^{4}-3781728 \omega_{3}^{2}+3548672$ \\
\hline$E_{6}=4\left(7488 \omega_{3}^{6}+278631 \omega_{3}^{4}-546104 \omega_{3}^{2}-2312448\right)$ \\
\hline$E_{7}=-4\left(13644 \omega_{3}^{6}-39301 \omega_{3}^{4}-1320541 \omega_{3}^{2}-787968\right)$ \\
\hline$E_{8}=-16\left(139 \omega_{3}^{6}+42559 \omega_{3}^{4}+113692 \omega_{3}^{2}+22016\right)$ \\
\hline$E_{9}=16\left(1569 \omega_{3}^{6}+11422 \omega_{3}^{4}+14765 \omega_{3}^{2}+768\right)$ \\
\hline$E_{10}=-128 \omega_{3}^{2}\left(39 \omega_{3}^{4}+132 \omega_{3}^{2}+101\right)$ \\
\hline$E_{11}=256 \omega_{3}^{2}\left(\omega_{3}^{2}+1\right)^{2}$ \\
\hline$F_{1}=-6377292 \omega_{3}^{2}$ \\
\hline$F_{2}=59049\left(19 \omega_{3}^{4}+1384 \omega_{3}^{2}+256\right)$ \\
\hline$F_{3}=-6561\left(9 \omega_{3}^{6}+2422 \omega_{3}^{4}+55404 \omega_{3}^{2}+29952\right)$ \\
\hline$F_{4}=729\left(1233 \omega_{3}^{6}+103915 \omega_{3}^{4}+870080 \omega_{3}^{2}+1108992\right)$ \\
\hline$F_{5}=-81\left(81 \omega_{3}^{8}+56709 \omega_{3}^{6}+1594980 \omega_{3}^{4}+4398976 \omega_{3}^{2}+13969408\right)$ \\
\hline$F_{6}=9\left(6561 \omega_{3}^{8}+910845 \omega_{3}^{6}+3240656 \omega_{3}^{4}+16711296 \omega_{3}^{2}+33681408\right)$ \\
\hline$F_{7}=-4\left(34992 \omega_{3}^{8}+244863 \omega_{3}^{6}-6689052 \omega_{3}^{4}+4233212 \omega_{3}^{2}-1824768\right)$ \\
\hline$F_{8}=4\left(5004 \omega_{3}^{8}-713205 \omega_{3}^{6}-3079245 \omega_{3}^{4}-1220800 \omega_{3}^{2}-1990656\right)$ \\
\hline$F_{9}=16\left(3411 \omega_{3}^{8}+64155 \omega_{3}^{6}+145780 \omega_{3}^{4}+27204 \omega_{3}^{2}+27648\right)$ \\
\hline$F_{10}=-16 \omega_{3}^{2}\left(1249 \omega_{3}^{6}+12350 \omega_{3}^{4}+15789 \omega_{3}^{2}-9440\right)$ \\
\hline$F_{11}=128 \omega_{3}^{2}\left(31 \omega_{3}^{6}+140 \omega_{3}^{4}+93 \omega_{3}^{2}-88\right)$ \\
\hline$F_{12}=-256 \omega_{3}^{4}\left(\omega_{3}^{2}+1\right)^{2}$ \\
\hline$T_{1}=729\left(3 \omega_{3}^{2}-8\right)$ \\
\hline$T_{2}=-324\left(9 \omega_{3}^{2}-91\right)$ \\
\hline$T_{3}=-144\left(75 \omega_{3}^{2}+437\right)$ \\
\hline$T_{4}=12\left(303 \omega_{3}^{2}-1124\right)$ \\
\hline$T_{5}=64\left(66 \omega_{3}^{2}+161\right)$ \\
\hline$T_{6}=-16\left(65 \omega_{3}^{2}+93\right)$ \\
\hline$T_{7}=64\left(\omega_{3}^{2}+1\right)$ \\
\hline$R=501$ \\
\hline
\end{tabular}

Table 11 Resonance associated to $\mathbf{k}^{11}$. Part III (continuation of Table 10): Terms appearing in Table 9, that contain the coefficients of the normal form $\mathcal{H}_{4}$ given in (19). 


\begin{tabular}{|l|l|}
\hline$c_{200}$ & $\frac{\left(4-\omega_{1}^{2}\right)\left(4-\omega_{1}^{2} \omega_{3}^{2}\right) \sum_{i=1}^{5} A_{i} \omega_{1}^{10-2 i}}{192\left(1-\omega_{3}^{2}\right)\left(\omega_{1}^{2}-\omega_{3}^{2}\right)^{2}\left(4 \omega_{1}^{2}-\omega_{3}^{2}\right)\left(4-\omega_{1}^{2} \omega_{3}^{2}\right)^{2}}$ \\
\hline$c_{020}$ & $\frac{\left(4-\omega_{3}^{2}\right)\left(4-\omega_{1}^{2} \omega_{3}^{2}\right) \sum_{i=1}^{5} B_{i} \omega_{1}^{10-2 i}}{576\left(1-\omega_{1}^{2}\right) \omega_{3}^{2}\left(\omega_{1}^{2}-\omega_{3}^{2}\right)^{2}\left(4-\omega_{1}^{2} \omega_{3}^{2}\right)^{2}}$ \\
\hline$c_{002}$ & $\frac{\left(4-\omega_{1}^{2}\right)\left(4-\omega_{3}^{2}\right) \sum_{i=1}^{3} C_{i} \omega_{1}^{6-2 i}}{12 \omega_{3}^{2}\left(4-\omega_{1}^{2} \omega_{3}^{2}\right)^{2}\left(\left(\omega_{3}^{2}-4\right) \omega_{1}^{2}-4 \omega_{3}^{2}+4\right)}$ \\
\hline$c_{110}$ & $\frac{\left(4-\omega_{1}^{2} \omega_{3}^{2}\right) \sum_{i=1}^{6} D_{i} \omega_{1}^{12-2 i}}{48 \omega_{1} \omega_{3}\left(\omega_{1}^{2}-\omega_{3}^{2}\right)^{2}\left(\omega_{1}^{2}-4 \omega_{3}^{2}\right)\left(4-\omega_{1}^{2} \omega_{3}^{2}\right)^{2}}$ \\
\hline$c_{011}$ & $\frac{\left(4-\omega_{1}^{2} \omega_{3}^{2}\right) \sum_{i=1}^{6} E_{i} \omega_{1}^{12-2 i}}{72 \omega_{1}^{2} \omega_{3}^{2}\left(\omega_{1}^{2}-\omega_{3}^{2}\right)\left(\omega_{1}^{2}-4 \omega_{3}^{2}\right)\left(4-\omega_{1}^{2} \omega_{3}^{2}\right)^{2}\left(\left(\omega_{3}^{2}-4\right) \omega_{1}^{2}-4 \omega_{3}^{2}+4\right)}$ \\
\hline$c_{101}$ & $\frac{\left(4-\omega_{1}^{2} \omega_{3}^{2}\right) \sum_{i=1}^{7} F_{i} \omega_{1}^{14-2 i}}{24 \omega_{1} \omega_{3}\left(\omega_{1}^{2}-\omega_{3}^{2}\right)\left(\omega_{1}^{2}-4 \omega_{3}^{2}\right)\left(4 \omega_{1}^{2}-\omega_{3}^{2}\right)\left(4-\omega_{1}^{2} \omega_{3}^{2}\right)^{2}\left(\left(\omega_{3}^{2}-4\right) \omega_{1}^{2}-4 \omega_{3}^{2}+4\right)}$ \\
\hline$\gamma_{3}$ & $\frac{\sum_{i=1}^{5} T_{i} \omega_{1}^{10-2 i}}{48 \omega_{1}^{2} \omega_{3}^{2}\left(\omega_{1}^{2}-\omega_{3}^{2}\right)\left(4-\omega_{1}^{2} \omega_{3}^{2}\right)\left(\left(\omega_{3}^{2}-4\right) \omega_{1}^{2}-4 \omega_{3}^{2}+4\right)}$ \\
\hline
\end{tabular}

Table 12 Resonance related to $\mathbf{k}^{12}$. Part I: Coefficients of the normal form $\mathcal{H}_{4}(\mathbf{I})$ given in (18). 


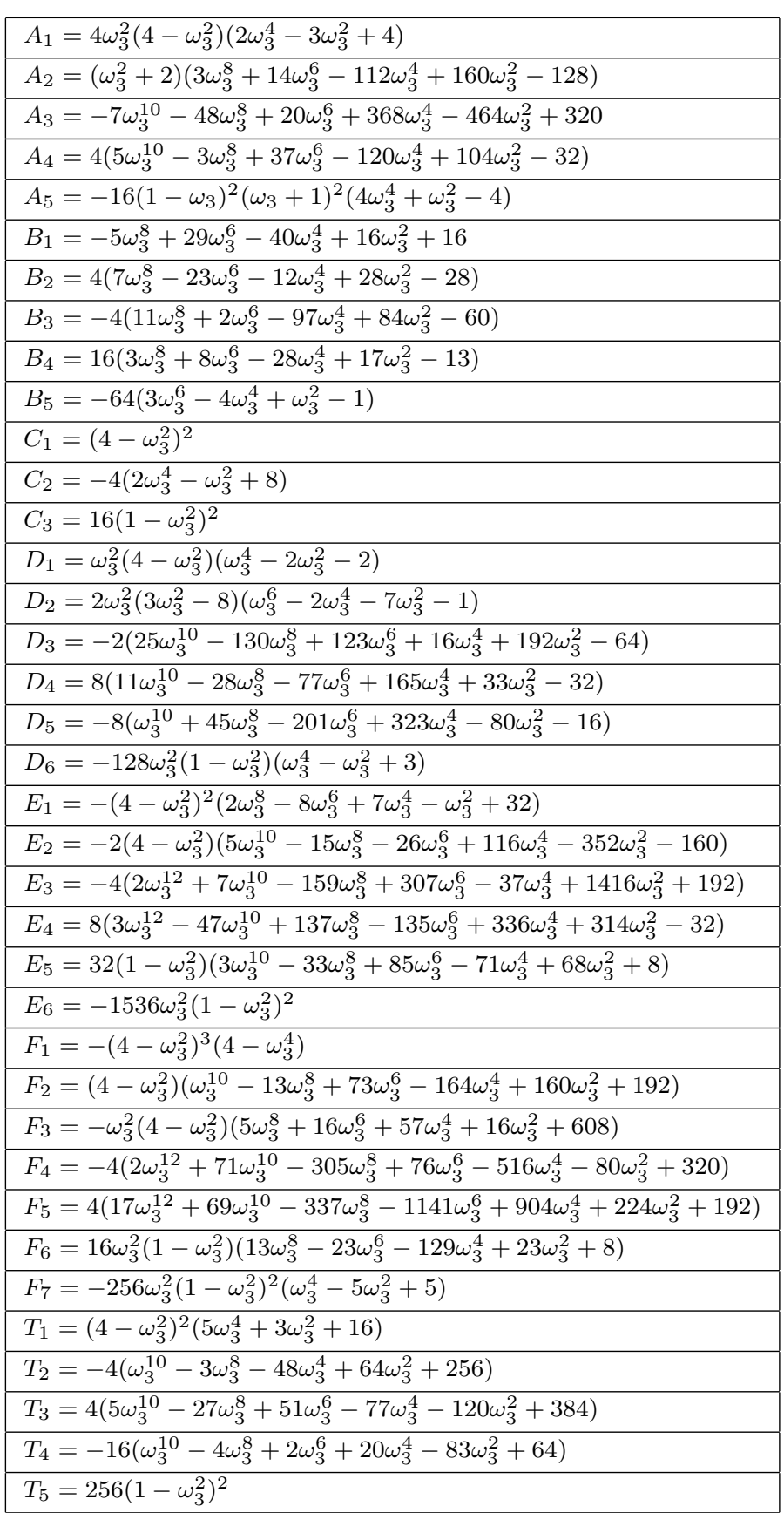

Table 13 Resonance associated to $\mathbf{k}^{12}$. Part II: Terms appearing in Table 12, which contain the coefficients of $\mathcal{H}_{4}(\mathbf{I})$ given in (18). 
Tables 14 and 15 contain the resonant cases of orders 3 and 4 corresponding to $\operatorname{dim} S=0$ and $\operatorname{dim} S=1$, respectively, in the absence of further resonances. The generic normal form (11) is not valid in these cases and then, a specific normal form is calculated in each situation.

\begin{tabular}{|l|l|l|l|}
\hline $\mathbf{k}^{i}$ & $F_{1}$ & $F_{2}$ & $\sigma=\left(\sigma_{1}, \sigma_{2}\right)$ \\
\hline $\mathbf{k}^{4}=(1,-1,-1)$ & $I_{1}+I_{3}$ & $I_{1}+I_{2}$ & $\left(\omega_{3},-\omega_{2}\right)$ \\
\hline $\mathbf{k}^{6}=(1,1,-1)$ & $I_{1}+I_{3}$ & $I_{2}-I_{1}$ & $\left(\omega_{3},-\omega_{2}\right)$ \\
\hline $\mathbf{k}^{7}=(2,0,-1)$ & $I_{1}+2 I_{3}$ & $I_{2}$ & $\left(\frac{\omega_{3}}{2},-\omega_{2}\right)$ \\
\hline $\mathbf{k}^{14}=(1,-1,-2)$ & $2 I_{1}+I_{3}$ & $I_{1}+I_{2}$ & $\left(\omega_{3},-\omega_{2}\right)$ \\
\hline $\mathbf{k}^{16}=(1,1,-2)$ & $2 I_{1}+I_{3}$ & $I_{2}-I_{1}$ & $\left(\omega_{3},-\omega_{2}\right)$ \\
\hline $\mathbf{k}^{21}=(2,0,-2)$ & $I_{1}+I_{3}$ & $I_{2}$ & $\left(\omega_{3},-\omega_{2}\right)$ \\
\hline
\end{tabular}

Table 14 Cases of $\operatorname{dim} S=0$ (in the absence of other resonances): resonance vectors $\mathbf{k}^{i}$ of orders 3 and 4 where the normal form (11) is not defined. The second and third columns contain the corresponding first integrals $F_{i}$ and the last column accounts for $\sigma$ such that $H_{2}=\sigma_{1} F_{1}+\sigma_{2} F_{2}$.

\begin{tabular}{|l|l|l|l|}
\hline $\mathbf{k}^{i}$ & $F_{1}$ & $F_{2}$ & $\sigma=\left(\sigma_{1}, \sigma_{2}\right)$ \\
\hline $\mathbf{k}^{2}=(0,2,1)$ & $I_{1}$ & $I_{2}-2 I_{3}$ & $\left(\omega_{1},-\omega_{2}\right)$ \\
\hline $\mathbf{k}^{11}=(1,3,0)$ & $I_{3}$ & $I_{2}-3 I_{1}$ & $\left(\omega_{3},-\omega_{2}\right)$ \\
\hline $\mathbf{k}^{12}=(0,2,2)$ & $I_{3}-I_{2}$ & $I_{1}$ & $\left(\omega_{3}, \omega_{1}\right)$ \\
\hline
\end{tabular}$?$

Table 15 Cases of $\operatorname{dim} S=1$ (in the absence of other resonances): resonance vectors $\mathbf{k}^{i}$ of orders 3 and 4 . The second and third columns contain the corresponding first integrals $F_{i}$ and the last column accounts for $\sigma$ such that $H_{2}=\sigma_{1} F_{1}+\sigma_{2} F_{2}$.

In Tables 16 and 17 we list the coefficients of the normalised Hamiltonian for two examples where KAM tori have been obtained in Sect. 5. The coefficients are represented in floating-point arithmetic but they have to be considered as approximations of the rational arithmetic computations performed with MathematicA.

\begin{tabular}{|l|l|l|l|}
\hline$c_{200}$ & 0.20358793 & $c_{110}$ & -0.38139098 \\
\hline$c_{020}$ & 0.09722169 & $c_{101}$ & -1.43131143 \\
\hline$c_{011}$ & 0.39138989 & $c_{002}$ & -0.25200856 \\
\hline
\end{tabular}

Table 16 KAM tori: Coefficients of the truncated normalised Hamiltonian function in the case of Remark 9. 


\begin{tabular}{|l|l|l|l|}
\hline$c_{200}$ & 0.14408762 & $c_{110}$ & 0.09681382 \\
\hline$c_{020}$ & 0.06550810 & $c_{101}$ & 0.64750232 \\
\hline$c_{011}$ & -0.19670504 & $c_{002}$ & -0.2613095 \\
\hline$d$ & -0.60105529 & \\
\hline
\end{tabular}

Table 17 KAM tori: Coefficients of the truncated normalised Hamiltonian function (22).

\section{Appendix: Nekhoroshev stability}

In the next paragraphs we apply Nekhoroshev stability results [28] to Hamiltonian (10) in the generic (non-resonant) case, i.e. with $\mathcal{H}_{4}$ given in (11). In this way we will be able to establish a comparison of the results obtained both by the Lie stability analysis and Nekhoroshev approach. For the verification of the steepness of $\mathcal{H}^{4}(\mathbf{I})$ we apply the algorithm given by Schirinzi and Guzzo in [29].

Denote by $\Lambda$ the 2 -dimensional linear space orthogonal to the linear space generated by the frequency vector $\omega$. Notice that the space $\Lambda$ plays the role of the set $S$ when $\operatorname{dim} S=2$.

Perform a rotation of $\mathbf{I}$ in order to carry $\omega$ to the first coordinate axis. The corresponding rotation matrix is

$$
R=\frac{1}{|\omega|}\left(\begin{array}{ccc}
\omega_{1} & -\omega_{2} & \omega_{3} \\
\omega_{2} & \frac{\omega_{1} \omega_{2}^{2}+\omega_{3}^{2}|\omega|}{\omega_{2}^{2}+\omega_{3}^{2}} & \frac{\left(|\omega|-\omega_{1}\right) \omega_{2} \omega_{3}}{\omega_{2}^{2}+\omega_{3}^{2}} \\
-\omega_{3} \frac{\left(|\omega|-\omega_{1} \omega_{2} \omega_{3}\right.}{\omega_{2}^{2}+\omega_{3}^{2}} & \frac{\omega_{2}^{2}|\omega| \omega_{1} \omega_{3}^{2}}{\omega_{2}^{2}+\omega_{3}^{2}}
\end{array}\right) .
$$

Denote by $\mathcal{A}$ the Hessian matrix associated to $\mathcal{H}_{4}(\mathbf{I})$, that is,

$$
\mathcal{A}=\left(\begin{array}{ccc}
2 c_{200} & c_{110} & c_{101} \\
c_{110} & 2 c_{020} & c_{011} \\
c_{101} & c_{011} & 2 c_{002}
\end{array}\right)
$$

and by $\mathcal{A}_{\Lambda}$ the restriction of the Hessian matrix to the space $\Lambda$, which is a $2 \times 2$-submatrix of $R \mathcal{A} R^{T}$. Precisely, $\mathcal{A}_{\Lambda}$ is the first second-order principal submatrix of $R \mathcal{A} R^{T}$, i.e. the matrix that results from eliminating the first row and the first column in matrix $R \mathcal{A} R^{T}$. Denote by $\lambda_{1} \equiv \lambda_{1}(C, A), \lambda_{2} \equiv \lambda_{2}(C, A)$ the eigenvalues of $\mathcal{A}_{\Lambda}$. They have the same sign in the red region shown in Fig. 9. Thus, $\mathcal{H}^{4}(\mathbf{I})$ is quasi-convex at the origin in this region. Applying the results in [5] and references therein, we conclude the following:

Theorem 8 Let $W_{1}=\left\{(C, A): \lambda_{1}>0, \lambda_{2}>0\right\}$ and $W_{2}=\left\{(C, A): \lambda_{1}<\right.$ $\left.0, \lambda_{2}<0\right\}$. Hamiltonian (10) truncated at order 2 is quasi-convex at the origin in region $W=W_{1} \cup W_{2}$. Then, for $\epsilon$ sufficiently small, any motion of Hamiltonian system related to (3) with initial conditions $|\mathbf{I}(0)| \leq \epsilon$ satisfies

$$
|\mathbf{I}(t)| \leq \epsilon^{1 / 3}, \quad \text { with } \quad|t| \leq \exp \left(\epsilon^{-1 / 3}\right)
$$


as well as

$$
|\mathbf{I}(t)| \leq \epsilon^{1 / 2}, \quad \text { with } \quad|t| \leq \exp \left(\epsilon^{-1 / 6}\right)
$$

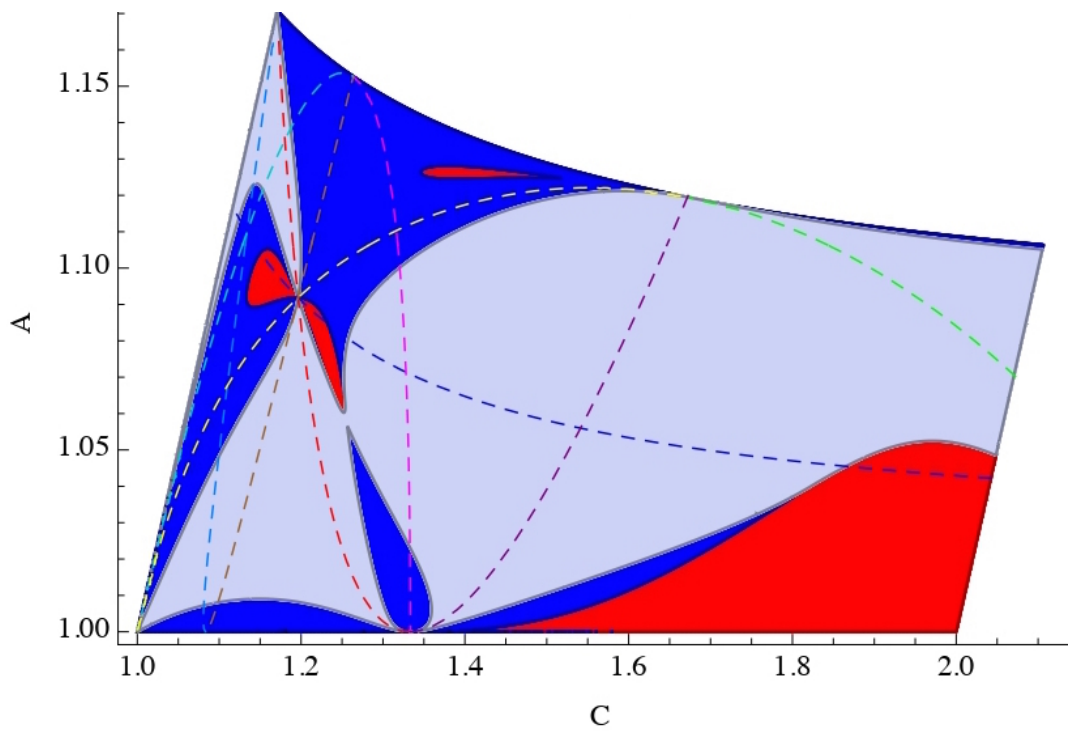

Fig. 9 Regions where Hamiltonian (10) is quasi-convex (red region) and directionally quasiconvex (dark blue region). Dashed colour lines account for the nine resonance curves of orders three and four.

Now, consider Hamiltonian (10) is not quasi-convex at the origin. The eigenvalues satisfy $\lambda_{1}<0<\lambda_{2}$. Denote by $\mathbf{v}_{1}, \mathbf{v}_{2}$ the eigenvectors of matrix $\mathcal{A}_{\Lambda}$ associated to $\lambda_{1}$ and $\lambda_{2}$, respectively. Then, construct

$$
\begin{aligned}
\mathbf{v}^{ \pm} & \equiv\left(v_{1}^{ \pm}, v_{2}^{ \pm}, v_{3}^{ \pm}\right) \\
& =R^{T}\left(0, \sqrt{\frac{\lambda_{2}}{\lambda_{2}-\lambda_{1}}} x_{1} \pm \sqrt{\frac{-\lambda_{1}}{\lambda_{2}-\lambda_{1}}} y_{1}, \sqrt{\frac{\lambda_{2}}{\lambda_{2}-\lambda_{1}}} x_{2} \pm \sqrt{\frac{-\lambda_{1}}{\lambda_{2}-\lambda_{1}}} y_{2}\right),
\end{aligned}
$$

where $\mathbf{x}=\left(x_{1}, x_{2}\right)=\frac{\mathbf{v}_{1}}{\left|\mathbf{v}_{1}\right|}$ and $\mathbf{y}=\left(y_{1}, y_{2}\right)=\frac{\mathbf{v}_{2}}{\left|\mathbf{v}_{2}\right|}$. Let

$$
\begin{aligned}
& V_{1}^{ \pm}=\left\{(C, A): v_{1}^{ \pm}>0, v_{2}^{ \pm}>0, v_{3}^{ \pm}<0\right\}, \\
& V_{2}^{ \pm}=\left\{(C, A): v_{1}^{ \pm}>0, v_{2}^{ \pm}<0, v_{3}^{ \pm}>0\right\}, \\
& V_{3}^{ \pm}=\left\{(C, A): v_{1}^{ \pm}<0, v_{2}^{ \pm}>0, v_{3}^{ \pm}>0\right\}, \\
& V_{4}^{ \pm}=\left\{(C, A): v_{1}^{ \pm}<0, v_{2}^{ \pm}<0, v_{3}^{ \pm}>0\right\}, \\
& V_{5}^{ \pm}=\left\{(C, A): v_{1}^{ \pm}<0, v_{2}^{ \pm}>0, v_{3}^{ \pm}<0\right\}, \\
& V_{6}^{ \pm}=\left\{(C, A): v_{1}^{ \pm}>0, v_{2}^{ \pm}<0, v_{3}^{ \pm}<0\right\} .
\end{aligned}
$$


Hamiltonian $\mathcal{H}_{4}(\mathbf{I})$ is directionally quasi-convex in the regions where the components of both vectors $\mathbf{v}^{ \pm}$change sign. This corresponds to the dark blue regions in Fig. 9. Then, by applying Theorem 1 in [5] we achieve the following:

Theorem 9 Let $V=\bigcup_{i=1}^{6} V_{i}^{+} \cap \bigcup_{i=1}^{6} V_{i}^{-}$. Hamiltonian (10) truncated at order 2 is directionally quasi-convex at the origin. Then, for $\epsilon$ sufficiently small, any motion of Hamiltonian system related to (3) with initial conditions such that $|\mathbf{I}(0)| \leq \epsilon$ satisfies the estimates

$$
|\mathbf{I}(t)| \leq \epsilon^{1 / 3}, \quad|t| \leq \exp \left(\epsilon^{-1 / 3}\right)
$$

as well as

$$
|\mathbf{I}(t)| \leq \epsilon^{1 / 2}, \quad|t| \leq \exp \left(\epsilon^{-1 / 6}\right)
$$

In summary, in II we obtain quasi-convexity regions (i.e. $W$ ) and directional quasi-convexity ones (i.e. $V$ ). To get insight in the light-blue region of Fig. 9, the 3-jet non-degeneracy should be studied. We stress that the quasi-convexity region (red area in Fig. 9), together with the directional quasi-convexity region (dark-blue region in Fig. 9), correspond to the Lie stability region (dark-blue region in Fig. 4) for any $\bar{\omega}_{1}$ and $\bar{\omega}_{2}$.

Acknowledgements The present paper is part of the thesis of the first author [6]. We thank Dr. Boris Bardin for his guidance and for sharing with us his expertise on this problem in the classical literature. We acknowledge Drs. Jair Koiller, Víctor Lanchares and Francisco Crespo for suggestions and comments in their review of this work as part of the thesis of D.C.-D. [6]. The help of Jhon Vidarte in the initial stage of approaching this problem is acknowledged.

\section{Conflict of interest}

The authors declare that they have no conflict of interest.

\section{References}

1. V.I. Arnold, V.V. Kozlov, A.I. Neishtadt, Mathematical Aspects of Classical and Celestial Mechanics, 3rd edition. Dynamical Systems III Encyclopaedia of Mathematical Sciences 3, Springer-Verlag, Berlin (2006)

2. B.S. Bardin, E.A. Chekina, On the stability of resonant rotation of a symmetric satellite in an elliptical orbit, Regul. Chaotic Dyn., 21(4), 377-389 (2016)

3. V.V. Beletskii, Motion of an Artificial Satellite about its Center of Mass, in Artificial Earth Satellites 1, pp. 25-43 Academy of Sciences, USSR (1958); in English: Artificial Earth Satellites. vols. 1 and 2, L.V. Kurnosova, Ed., pp. 30-54, Plenum Press, New York (1960)

4. V.V. Beletskii, Motion of an Artificial Satellite about its Center of Mass. Series: Mechanics of Space Flight, translated from Russian, published for the National Aeronautics and Space Administration, U.S.A. and the National Science Foundation, Washington, D.C. by the Israel Program for Scientific Translations, Jerusalem (1966)

5. G. Benettin, F. Fassò, M. Guzzo, Nekhoroshev-stability of $L_{4}$ and $L_{5}$ in the spatial restricted three-body problem, Regul. Chaotic Dyn., 3(3), 56-70 (1998) 
6. D. Cárcamo-Díaz, Stability and estimates near elliptic equilibrium points in Hamiltonian systems and applications, PhD Thesis, Universidad del Bío-Bío, Concepción, Chile (2019)

7. D. Cárcamo-Díaz, J.F. Palacián, C. Vidal, P. Yanguas, Nonlinear stability of elliptic equilibria in Hamiltonian systems with exponential time estimates, preprint (2019)

8. D. Cárcamo-Díaz, J.F. Palacián, C. Vidal, P. Yanguas, On the nonlinear stability of the triangular points in the circular spatial restricted three-body problem, Regul. Chaotic Dyn., 25(2), 131-148 (2020)

9. P. Chartier, A. Murua, J.M. Sanz-Serna, Higher-order averaging, formal series and numerical integration III: error bounds, Found. Comput. Math., 15(2), 591-612 (2015)

10. D.B. DeBra, R.H. Delp, Rigid body attitude stability and natural frequencies in a circular orbit, J. Astronaut. Sci., 8, 14-17 (1961)

11. R.H. Delp, Attitude Motion of a Small Satellite in an Inverse-Square Central-Force Field, Lockheed Missiles and Space Division, 417670 (1958)

12. A. Deprit, Canonical transformations depending on a small parameter, Celestial Mech., 1, 12-30 (1969)

13. G.L. Dirichlet, Über die stabilität des Gleichgewichts, J. Reine Angew. Math., 32, 85-88 (1846)

14. F. dos Santos, J.E. Mansilla, C. Vidal, Stability of equilibrium solutions of autonomous and periodic Hamiltonian systems with $n$ degrees of freedom in the case of single resonance, J. Dyn. Differ. Equ., 22(4), 805-821 (2010)

15. F. dos Santos, C. Vidal, Stability of equilibrium solutions of autonomous and periodic Hamiltonian systems in the case of multiple resonances, J. Differential Equations, 258(11), 3880-3901 (2015)

16. H.S. Dumas, K.R. Meyer, J.F. Palacián, P. Yanguas, Asymptotic stability estimates near an equilibrium point, J. Differential Equations, 263(2), 1125-1139 (2017)

17. C.D. Hall, Spacecraft Attitude Dynamics and Control, Lecture Notes posted on http://citeseerx.ist.psu.edu/viewdoc/download?doi=10.1.1.608.4249\&rep=rep1\&type=pdf (2000)

18. Y. Han, Y. Li, Y. Yi, Invariant tori in Hamiltonian systems with high order proper degeneracy, Ann. Henri Poincaré, 10(8), 1419-1436 (2010)

19. P.C. Hughes, Spacecraft Attitude Dynamics, Dover Publications, Inc., Mineola, New York (2004)

20. L.G. Khazin, On the stability of Hamiltonian systems in the presence of resonances, Prikl. Mat. Mekh., 35(3), 423-431 (1971); J. Appl. Math. Mech., 35(3), 384-391 (1971)

21. S.R. Marandi, V.J. Modi, Attitude stability of rigid satellites via a normalized Hamiltonian, Acta Astronaut., 19(4), 287-299 (1989)

22. A.P. Markeev, Libration Points in Celestial Mechanics and Astrodynamics, In Russian, Moscow Izdatel'stvo Nauka, Moscow (1978)

23. A.P. Markeev, Linear Hamiltonian Systems and Certain Problems of Stability of Motion of a Satellite in Relation to its Center of Mass, in Russian, NITs Regular and Chaotic Dynamics, Institute of Computational Analysis, Moscow-Izhevsk, Moscow (2009)

24. A.P. Markeev, A.G. Sokol'skii, On the stability of relative equilibrium of a satellite in a circular orbit, Kosmicheskie Issledovaniya, 13(2), 139-146 (1975); Cosm. Res., 13(2), $119-125(1975)$

25. K.R. Meyer, D. Offin, Introduction to Hamiltonian Dynamical Systems and the N-Body Problem, 3rd edition. Springer-Verlag, New York (2017)

26. K.R. Meyer, J.F. Palacián, P. Yanguas, Singular reduction of resonant Hamiltonians, Nonlinearity, 31, 2854-2894 (2018)

27. J. Moser, New aspects in the theory of stability of Hamiltonian systems, Comm. Pure Appl. Math., 11(1), 81-114 (1958)

28. N.N. Nekhoroshev, An exponential estimate of the time of stability of nearly-integrable Hamiltonian systems, Uspekhi Mat. Nauk., 32(6), 5-66 (1977); Russ. Math. Surv., 32(6), $1-65(1977)$

29. G. Schirinzi, M. Guzzo, Numerical verification of the steepness of three and four degrees of freedom Hamiltonian systems, Regul. Chaotic Dyn., 20(1), 1-18 (2015)

30. C.L. Siegel, Über die Existenz einer Normalform analytischer Hamiltonscher Differential Gleichungen in der Nähe einer Gleichgewichtslösung, Math. Ann., 128, 144-170 (1954) 
31. V.E. Zhavnerchik, On the stability of autonomous systems in the presence of several resonances, Prikl. Mat. Mekh., 43(2), 229-234 (1979); J. Appl. Math. Mech., 43(2), 247253 (1979)

32. V.E. Zhavnerchik, On stability in the presence of multiple resonance of odd order, Prikl. Mat. Mekh., 44(6), 971-976 (1980); J. Appl. Math. Mech., 44(6), 692-696 (1980) 